\title{
Optimal Consumption and Portfolio Selection with Stochastic Differential Utility ${ }^{1}$
}

\author{
Mark Schroder
}
Department of Finance of the Eli Broad Graduate School of Management, Michigan State University, East Lansing, Michigan 48824
schrode7@pilot.msu.edu

and

\section{Costis Skiadas}

\begin{abstract}
Department of Finance of the J. L. Kellogg Graduate School of Management, Northwestern University, Evanston, Illinois 60208 c-skiadas@nwu.edu
\end{abstract}

Received November 21, 1997; revised May 19, 1999

We develop the utility gradient (or martingale) approach for computing portfolio and consumption plans that maximize stochastic differential utility (SDU), a continuous-time version of recursive utility due to D. Duffie and L. Epstein (1992, Econometrica 60, 353-394). We characterize the first-order conditions of optimality as a system of forward-backward SDEs, which, in the Markovian case, reduces to a system of PDEs and forward only SDEs that is amenable to numerical computation. Another contribution is a proof of existence, uniqueness, and basic properties for a parametric class of homothetic SDUs that can be thought of as a continuoustime version of the CES Kreps-Porteus utilities studied by L. Epstein and A. Zin (1989, Econometrica 57, 937-969). For this class, we derive closed-form solutions in terms of a single backward SDE (without imposing a Markovian structure). We conclude with several tractable concrete examples involving the type of "affine" state price dynamics that are familiar from the term structure literature. Journal of Economic Literature Classification Numbers: G11, E21, D91, D81, C61. (C) 1999 Academic Press

${ }^{1}$ We are grateful for helpful discussions with John Campbell, George Constantinides, Kent Daniel, Darrell Duffie, Bernard Dumas, Janice Eberly, Mark Fisher, John Heaton, Ravi Jagannathan, Erzo Luttmer, Lars Nielsen, Ken Singleton, Raman Uppal, Luis Viceira, and Tan Wang. We are responsible for any errors. Results omitted in the published version can be downloaded from http://www.kellogg.nwu.edu/faculty/skiadas/research/research.htm. 


\section{INTRODUCTION}

This paper develops the utility gradient, or martingale, approach for solving optimal consumption-portfolio selection problems in continuoustime complete markets with Brownian information and stochastic differential utility (SDU). SDU was introduced by Duffie and Epstein [12] as a continuous time limit of the type of recursive utility studied by Kreps and Porteus [35], Epstein and Zin [24], and many others.

The importance and structure of preferences that are not necessarily temporally additive have been studied extensively in a literature surveyed by Epstein [23]. It is, for example, well known that utility additivity with respect to time and states of nature is overly restrictive in expressing reasonable notions of risk aversion in temporal settings. (The introduction of Duffie and Epstein [12] gives a suggestive example.) As Epstein [23] explains in his survey, under additive temporal preferences notions of intertemporal substitution and risk aversion are inflexibly linked to each other. SDU, while nesting the time-additive case, is more flexible in this regard, capturing the notion that one's present sense of well-being can depend on one's expected future utility levels in a not necessarily riskneutral manner. This effect is also related to attitudes toward the timing of resolution of uncertainty, as discussed, among others, by Kreps and Porteus [35], Chew and Epstein [3,4], and Skiadas [47], the last reference covering the case of SDU.

The paper's approach generalizes the Karatzas et al. [31], and Cox and Huang [5,6] treatments ${ }^{2}$ of Merton's [40] optimal portfolio selection problem with additive utilities (textbook accounts of which are given by Merton [41], Duffie [11], and Karatzas and Shreve [33]). The basic idea is to utilize market completeness to separate the computation of an optimal consumption plan and that of a corresponding trading strategy. The optimal consumption is obtained by solving the first-order conditions, essentially stating that the agent's marginal utility process at the optimum is proportional to an Arrow-Debreu state price density process. A corresponding financing trading strategy can then be constructed using Merton's standard replication arguments (developed in the context of the BlackScholes theory of option pricing). Duffie and Skiadas [20] showed that this approach remains valid quite generally, even with non-additive preferences, provided that a marginal utility process is well defined as the Riesz representation of the (infinite-dimensional) utility gradient at the optimum. Moreover, they obtained closed-form expressions for marginal utilities in this sense for various types of temporally dependent preferences used in practice, including SDU. Even earlier, Duffie and Epstein [12]

${ }^{2}$ Closely related are the works of Pliska [44] and Foldes [26]. 
used dynamic programming methods to compute the form of ArrowDebreu prices under SDU in a more special Markovian setting under Brownian information. The Duffie-Epstein-Skiadas papers, however, are oriented toward obtaining equilibrium pricing formulas and do not in fact solve the first-order conditions for the optimal consumption plan.

With additive utilities, the solution of the first-order conditions amounts to a straightforward inversion of the marginal utility at each state-time separately. With SDU, the solution of the first-order conditions involves the solution of a system of so-called forward-backward stochastic differential equations (FBSDEs), a fixed-point problem involving the complete information filtration at once. The mathematical theory of FBSDEs is fairly recent, and a satisfactorily general existence theory is missing at the moment. Ma et al. [39] have developed a useful scheme for solving FBSDEs in Markovian settings with Brownian information via the solution of quasilinear partial differential equations (PDEs). A first contribution of this paper is to import the methodology of Ma et al. to the problem of optimal portfolio selection in a Markovian setting with Brownian information. The result is a remarkably simple solution procedure that complements the more traditional dynamic programming approach and in fact results in a numerically more tractable PDE form than that associated with the Bellman equation. A similar approach applies with other temporally dependent utility forms whose gradients are computed by Duffie and Skiadas [20]. In order to keep this paper focused and of manageable size, we will restrict our analysis to the SDU case. Schroder and Skiadas [46] present extensions that include habit formation.

In addition to providing a general solution method, we will apply this approach to a parametric class of homothetic SDU that was introduced by Duffie and Epstein $[12,13]$ as a continuous-time limit of the CES Kreps-Porteus specification studied by Epstein and Zin [24] and by Weil [51]. Without Markovian assumptions, we will show that the first-order conditions simplify significantly in this case, resulting in closed-form expressions in terms of the solution to a single backward SDE. The latter becomes trivial under a deterministic investment opportunity set, while in more general Markovian settings the solution of this backward SDE reduces to the solution of a numerically tractable PDE. Analytic solutions will be derived for examples involving a stochastic investment opportunity set. A difficulty with this parametric SDU class is that both the utility specification and the associated first-order conditions involve backward SDEs that violate the usual Lipschitz growth assumptions of available existence results by Pardoux and Peng [43] and Duffie and Epstein [12]. Instead, we will prove existence, uniqueness, and basic properties from first principles using monotonicity arguments. Another difficulty is that most of 
the technical restrictions required by the results of Ma et al. [39] and Duffie and Skiadas [20] are also violated in this context. Again, we will provide the requisite special arguments that prove optimality of the proposed solutions.

For some of the utility parameter ranges we consider, Svensson [49] and Obstfeld [42] have provided heuristic derivations of the solutions for the special case of a constant (deterministic) investment opportunity set. They have not addressed, however, the issues of utility function existence and optimality verification. Fisher and Gilles [25] have also worked, concurrently with this paper $^{3}$, on a parametric homothetic case in an infinite horizon setting with a stochastic investment opportunity set. While their examples can be viewed as infinite horizon limits of examples we analyze in this paper, the two papers have different objectives. Fisher and Gilles manipulate the utility forms and first-order conditions heuristically and proceed quickly to numerical solutions of PDEs. In contrast, the present paper contains no numerical examples, but instead concentrates on the theoretical development, which, as noted above, includes the requisite backward SDE theory and optimality verification arguments.

Discrete time versions of some of our homothetic examples have been analyzed by Giovannini and Weil [29] and Campbell and Viceira [2]. The former concentrate on special cases resulting in myopic portfolios or consumption plans, and the latter use an approximate log-linearization of the budget constraint. Some special cases involving additive utility and specific parameterizations of price dynamics are closely related to those of Kim and Omberg [34], Liu [37], and Wachter [50] (the latter two were written concurrent with and independent of the present paper.) Finally, this paper is related to several other papers that discuss the use of FBSDEs in different contexts of finance theory, including Cvitanic [7]; Cvitanić and Ma [8]; Duffie et al. [14, 17, 18, 19]; and El Karoui et al. [22]. The last reference also discusses a dual characterization of SDU, introduced by Geoffard [28] (for the deterministic case) and used by Dumas et al. [21] in their study of efficient allocations with SDU.

The remainder of this paper is organized into six sections and three appendixes. Section 2 gives an abstract formulation of the problem,

\footnotetext{
${ }^{3}$ Fisher and Gilles originally used an early version of our paper that included the general solution method, but not the current Theorems 3 and 4 . They independently derived heuristic versions of these results for the infinite horizon case. Their discussion offers useful intuition and complements ours. They did not address, however, the questions of existence of the utility function being maximized and of the stochastic process used to describe the candidate optimal solution. Neither did they prove that the proposed solution is in fact optimal. More recent versions of their paper make full use of our results as reported here.
} 
presenting the first-order conditions of optimality in terms of Arrow-Debreu prices and the Riesz representation of the utility gradient. Section 3 shows how the general analysis applies with SDU and outlines a computational approach in Markovian settings. Section 4 introduces a parametric class of homothetic SDU, develops their basic properties, and presents optimal consumption and portfolio rules in closed form for several special cases. The general solution method for this SDU class is discussed in Section 5, followed in Section 6 by several examples with a stochastic investment opportunity set. Section 7 concludes with an outline of how to incorporate a bequest function in the analysis and a parametric example. The appendixes contain proofs, and they develop some requisite mathematical theory.

\section{ABSTRACT FORMULATION}

We begin with a probability space $(\Omega, \mathscr{F}, P)$ supporting a $n$-dimensional standard Brownian motion, $B$, over the finite time horizon $[0, T]$. All stochastic processes introduced in this paper will be assumed progressively measurable with respect to the augmented filtration $\left\{\mathscr{F}_{t}: t \in[0, T]\right\}$ generated by $B$. We also assume that $\mathscr{F}=\mathscr{F}_{T}$. The conditional expectation operator $E\left[\cdot \mid \mathscr{F}_{t}\right]$ will be abbreviated to $E_{t}$ throughout.

We let $D$ denote the Hilbert space containing any progressively measurable process of the form $x: \Omega \times[0, T] \rightarrow \mathbb{R}$ satisfying $E\left(\int_{0}^{T} x_{t}^{2} d t\right)$ $<\infty$. The inner product of $D$ is defined by $\langle x, y\rangle=E\left(\int_{0}^{T} x_{t} y_{t} d t\right)$. As usual, we identify any $x, y \in D$ such that $\langle x-y, x-y\rangle=0$. The space of all processes is partially ordered by letting $x \geqslant(>) y$ denote the condition: $x(\omega, t) \geqslant(>) y(\omega, t)$ for almost every $(\omega, t)$. The positive cone of $D$ is $D^{+}=\{x \in D: x \geqslant 0\}$, and the strictly positive cone is $D^{++}=$ $\{x \in D: x>0\}$. For mathematical background material we refer to Karatzas and Shreve [32].

\subsection{Optimality in an Arrow-Debreu Market}

We take as primitive a consumption space $\mathscr{C}$, assumed throughout to satisfy:

$\mathrm{C} 1 . \mathscr{C}$ is a cone that is a subset of $D^{+}$and has the property that for every bounded $h \in D^{+}$and $c \in \mathscr{C}, c+h \in \mathscr{C}$.

For example, we can take $\mathscr{C}=D^{+}$, but in our main application we will need to impose additional integrability conditions on elements of $\mathscr{C}$. A consumption process, $c$, is any element of $\mathscr{C}$, with $c_{t}$ representing a time $t$ consumption rate in terms of a single numeraire good. For simplicity of 
exposition, we do not allow terminal consumption, although our analysis extends in a straightforward manner to include it, as outlined in the concluding section.

We will consider an agent with initial wealth $w>0$ that maximizes a utility function $V_{0}: \mathscr{C} \rightarrow \mathbb{R}$ by trading in a complete securities market (that is, a market in which every consumption plan can be financed given sufficient initial wealth).

Given $c \in \mathscr{C}$, we let $F(c)$ denote the set of feasible directions at $c$, that is, the set of any $h \in D$ such that $c+\alpha h \in \mathscr{C}$ for all sufficiently small positive $\alpha$. The Gateaux derivative of $V_{0}$ at $c$ is defined by

$$
\nabla V_{0}(c ; h)=\lim _{\alpha \downarrow 0} \frac{V_{0}(c+\alpha h)-V_{0}(c)}{\alpha}, \quad h \in F(c) .
$$

This derivative is closely related to a function $m: \mathscr{C} \rightarrow D$, which we take as given throughout the paper, and, for SDU, will be given in closed form. We will use $m$ through one of two conditions. The first condition, satisfied under general assumptions on the utility form (see Duffie and Skiadas [20]), but not always, is

\section{C2. For every $c \in \mathscr{C}, \nabla V_{0}(c ; h)=\langle m(c), h\rangle$ for all $h \in F(c)$.}

In particular, $\mathrm{C} 2$ implies that, for every $c$, the Gateaux derivative of $V_{0}$ at $c$ exists and is linear. Under these conditions, $\nabla V_{0}(c, \cdot)$ is called the utility gradient of $V_{0}$ at $c$, with Riesz representation $m$.

In some applications, condition $\mathrm{C} 2$ is unnecessarily strong for the purpose of confirming optimality of a given consumption plan, and we will instead use the following inequalities, where $\hat{c} \in \mathscr{C}$ is some candidate optimal consumption plan:

$$
\mathrm{C} 2^{\prime} \text {. For all } c \in \mathscr{C}, V_{0}(c) \leqslant V_{0}(\hat{c})+\langle m(\hat{c}), c-\hat{c}\rangle .
$$

In complete markets, the determination of an optimal consumption plan depends only on preferences, endowments, and Arrow-Debreu state prices, while the corresponding financing strategy can be derived using standard replication arguments. An Arrow-Debreu state price density is any strictly positive process, $\pi \in D^{++}$, such that capital plus dividend gains from trade deflated by $\pi$ form a martingale. Bypassing, for now, the familiar derivation of state price dynamics from price dynamics, we take as primitive a (normalized) state price density process, $\pi$, with dynamics

$$
-\frac{d \pi_{t}}{\pi_{t}}=r_{t} d t+\eta_{t}^{\prime} d B_{t}, \quad \pi_{0}=1
$$


where $\int_{0}^{T}\left|r_{t}\right|+\eta_{t}^{\prime} \eta_{t} d t<\infty$ a.s. The process $r$ is the short-rate process, and $\eta$ is the market-price-of-risk process. ${ }^{4}$

The consumption process $\hat{c}$ is defined to be optimal if

$$
\hat{c} \in \arg \max \left\{V_{0}(c):\langle\pi, c\rangle \leqslant w, c \in \mathscr{C}\right\} .
$$

\subsection{First-Order Conditions}

Given $\mathrm{C} 2$, the first-order conditions for optimality of $c \in \mathscr{C}$ take the familiar form

$$
\begin{aligned}
& m_{t}(c)=\lambda \pi_{t} \quad \text { on } \quad\left\{c_{t}>0\right\} \quad \text { for a.e. } t \in[0, T] \text {, } \\
& m_{t}(c) \leqslant \lambda \pi_{t} \quad \text { on } \quad\left\{c_{t}=0\right\} \text { for a.e. } t \in[0, T] \text {, } \\
& \langle\pi, c\rangle \leqslant w, \quad \lambda(w-\langle\pi, c\rangle)=0, \quad \lambda \in \mathbb{R}^{+} .
\end{aligned}
$$

The specification "on" some event in (1), and throughout the paper, should be interpreted in the almost sure sense.

Proposition 1. (a) Suppose that conditions $\mathrm{C} 1$ and $\mathrm{C} 2$ hold. Then (1) holds for every optimal $c \in \mathscr{C}$. (b) Suppose that conditions $\mathrm{C} 1$ and $\mathrm{C} 2$ ' hold, for some $\hat{c} \in \mathscr{C}$. If (1) is satisfied with $c=\hat{c}$, then $\hat{c}$ is optimal. (c) If $V_{0}$ is concave and continuous and $\mathscr{C}$ is convex and closed, then an optimal consumption process exists.

Proof. (a) Consider the following first-order conditions for optimality of $c \in \mathscr{C}$ :

$$
\begin{aligned}
\nabla V_{0}(c ; h)-\lambda\langle\pi, h\rangle & \leqslant 0, \quad h \in F(c), \\
\langle\pi, c\rangle & \leqslant w, \quad \lambda(w-\langle\pi, c\rangle)=0, \quad \lambda \in \mathbb{R}^{+} .
\end{aligned}
$$

By the generalized Kuhn-Tucker theorem, these conditions are necessary for optimality of $c$. For example, Theorem 1 of Section 9.4 of Luenberger [38] can be used, with only a straightforward modification of the proof to account for the fact that the underlying space is a positive cone, and not a linear space. Given $\mathrm{C} 1$ and the utility gradient Riesz representation, $m$, the above conditions imply that for all bounded $h \in D^{+},\langle m(c)-\lambda \pi, h\rangle$ $\leqslant 0$, and therefore $m_{t}(c) \leqslant \lambda \pi_{t}$ a.s. for almost every $t$. Since we also have

\footnotetext{
${ }^{4}$ An equivalent formulation results if, instead of state prices, we consider an equivalent martingale measure, in the sense of Harrison and Kreps [30], relative to the short-rate process $r$. This, for example, is the approach taken by Cox and Huang [5]. For the purposes of this paper, the formulation in terms of state prices is notationally more parsimonious. A textbook exposition of the relationship between state prices and equivalent martingale measures can be found in Duffie [11].
} 
$\langle m(c)-\lambda \pi, \alpha c\rangle \leqslant 0$ for $\alpha>-1, m_{t}(c)=\lambda \pi_{t}$ on $\left\{c_{t}>0\right\}$ for almost every $t$. This proves part (a).

(b) If (1) holds with $c=\hat{c}$, then $\langle m(\hat{c}), c-\hat{c}\rangle \leqslant 0$ for any budget feasible $c \in \mathscr{C}$, and the result follows from the gradient inequality of condition $\mathrm{C}^{\prime}$.

(c) If $V_{0}$ is concave and norm-continuous, it is weakly upper-semicontinuous. By Alaoglu's theorem, and since for convex sets strong closure is the same as weak closure, the budget feasible set is weakly compact. The maximum is therefore achieved.

\subsection{Securities Market}

The above discussion is all in the setting of an Arrow-Debreu market. The implementation of the optimal consumption plan by trading in a securities market is well understood. In order to fix notation in discussing applications later on, we now outline a simple securities market that implements the above Arrow-Debreu market. More general formulations and details can be found in the expositions of Duffie [11] and Karatzas and Shreve [33].

In addition to short-term default-free borrowing and lending at a rate given by the process $r$, there are $n$ risky securities (one for each component of the Brownian motion $B$ ). The risky asset instantaneous excess returns (relative to $r$ ) are represented by the $n$-dimensional Ito process $R_{t}=$ $\left[R_{t}^{1}, \ldots, R_{t}^{n}\right]^{\prime}$, with decomposition

$$
d R_{t}=\mu_{t}^{R} d t+\sigma_{t}^{R} d B_{t},
$$

where $\mu^{R}$ and $\sigma^{R}$ are progressively measurable processes valued in $\mathbb{R}^{n}$ and $\mathbb{R}^{n \times n}$, respectively, and satisfy $\int_{0}^{T}\left|\mu_{t}^{R}\right|+\sigma_{t}^{R}\left(\sigma_{t}^{R}\right)^{\prime} d t<\infty$ a.s. We assume that $\sigma_{t}^{R}$ is invertible almost everywhere, and $\eta_{t}=\left(\sigma_{t}^{R}\right)^{-1} \mu_{t}^{R}$.

A trading strategy is any progressively measurable process, $\psi$, valued in $\mathbb{R}^{n}$, such that $\int_{0}^{t}\left|\psi_{s}^{\prime} \mu_{s}^{R}\right|+\psi_{s}^{\prime} \sigma_{s}^{R}\left(\sigma_{s}^{R}\right)^{\prime} \psi_{s} d s<\infty$ a.s. for all $t<T$. We interpret $\psi_{t}^{i}$ as the time $t$ proportion of wealth invested in security $i \in\{1, \ldots, n\}$, the remaining wealth being invested at the short rate $r$. Given any initial wealth $w$, consumption plan $c$, and trading strategy $\psi$, the corresponding wealth process $W^{w, c, \psi}$ is defined by the budget equation:

$$
d W_{t}^{w, c, \psi}=W_{t}^{w, c, \psi}\left(\psi_{t}^{\prime} d R_{t}+r_{t} d t\right)-c_{t} d t, \quad t<T, \quad W_{0}^{w, c, \psi}=w .
$$

We say that $\psi$ finances $c$ given initial wealth $w$ if $W_{t}^{w, c, \psi} \geqslant 0$ a.s. for all $t<T$.

For every consumption plan $c$, we define the process

$$
W_{t}(c)=\frac{1}{\pi_{t}} E_{t}\left[\int_{t}^{T} \pi_{s} c_{s} d s\right] .
$$


Recalling the normalization $\pi_{0}=1$, we have $W_{0}(c)=\langle\pi, c\rangle$. It can be shown (see, for example, Karatzas and Shreve [33]) that for every consumption plan $c$, there exists a trading strategy $\psi$ that finances $c$ given initial wealth $w=\langle\pi, c\rangle$, such that $W(c)=W^{w, c, \psi}$. This trading strategy can be calculated in terms of the wealth dynamics $d W_{t}(c) / W_{t}(c)=$ $\mu_{t}^{W(c)} d t+\sigma_{t}^{W(c)} d B_{t}$, by matching the diffusion term with that of the budget equation, to obtain $\psi=\left(\sigma_{t}^{R^{\prime}}\right)^{-1} \sigma_{t}^{W(c)^{\prime}}$. Conversely, if $c$ can be financed given some initial wealth $w$, then $\langle\pi, c\rangle \leqslant w$. These familiar results imply that the problem of determining an optimal trading strategy given initial wealth $w$ reduces to the optimal consumption problem of Section 2.1.

\section{SOLUTION METHOD WITH STOCHASTIC DIFFERENTIAL UTILITY}

The general approach of the last section is further developed in this section for the special case of stochastic differential utility.

\subsection{Stochastic Differential Utility}

Stochastic differential utility is defined in terms of a function $f:[0, T] \times$ $\mathbb{R}^{+} \times \mathbb{R} \rightarrow \mathbb{R}$, called the intertemporal aggregator. We refer to the three arguments of $f$ as the time, consumption, and utility argument, respectively, and we write $f_{c}$ and $f_{v}$ for the partial derivatives of $f$ with respect to the consumption and utility arguments, respectively. To every $c \in \mathscr{C}$ we assign a utility process, $V(c)$, that satisfies

$$
V_{t}(c)=E_{t}\left[\int_{t}^{T} f\left(s, c_{s}, V_{s}(c)\right) d s\right], \quad t \in[0, T] .
$$

The following condition will be assumed throughout:

C3. There exists $\mathscr{V} \subseteq D$ such that, for every $c \in \mathscr{C}$, a unique $V(c) \in \mathscr{V}$ satisfies (2). The utility function $V_{0}: \mathscr{C} \rightarrow \mathbb{R}$ is defined by the initial values of $V(c) \in \mathscr{V}, c \in \mathscr{C}$, and the function $m: \mathscr{C} \rightarrow D$ (appearing in $\mathrm{C} 2$ or $\mathrm{C} 2{ }^{\prime}$ ) is given by

$$
m_{t}(c)=\exp \left(\int_{0}^{t} f_{v}\left(s, c_{s}, V_{s}(c)\right) d s\right) f_{c}\left(t, c_{t}, V_{t}(c)\right) .
$$

The combined results of Duffie and Epstein [12] and Duffie and Skiadas [20] show that conditions $\mathrm{C} 2$ and $\mathrm{C} 3$ (with $\mathscr{C}=D^{+}$and $\mathscr{V}=D$ ) hold if $f$ satisfies certain continuity-Lipschitz-growth type conditions. ${ }^{5}$ Moreover,

\footnotetext{
${ }^{5}$ More specifically, it is sufficient that $f$ be continuously differentiable in its consumption and utility arguments, and for some constant $K,|f(t, c, 0)| \leqslant K(1+c), \quad\left|f_{c}(t, c, v)\right| \leqslant$ $K(1+c+|v|)$, and $\left|f_{v}(t, c, v)\right| \leqslant K$, for all $t \in[0, T], c \in \mathbb{R}^{+}$, and $v \in \mathbb{R}$.
} 
under the same restrictions on $f, V_{0}$ is continuous, $V_{0}$ is increasing if $f$ is increasing in its consumption argument, and $V_{0}$ is concave if $f$ is concave jointly in its consumption and utility arguments.

Unfortunately, the conditions on $f$ imposed by the Duffie-EpsteinSkiadas results are violated in the parametric cases discussed later in this paper. We will therefore present the general solution method by directly assuming the validity of conditions $\mathrm{C} 1, \mathrm{C} 2$ or $\mathrm{C} 2$, and $\mathrm{C} 3$, as well as the following restriction on $f$ whose purpose is to simplify the first-order conditions for optimality:

C4. For all $t \in[0, T], \quad c \in \mathbb{R}^{+}$, and $v \in \mathbb{R}, \quad f_{c}(t, c, v)>0$ and $\lim _{x \rightarrow \infty} f_{c}(t, x, v)=0$.

In applications, the above conditions will be verified on a case-by-case basis.

For the case of a concave intertemporal aggregator, the following result provides a direct justification for the expression (3) and an easy way of confirming $\mathrm{C}^{\prime}$, and hence the sufficiency of the first-order conditions for optimality.

Lemma 1. Suppose that condition $\mathrm{C} 3$ is satisfied, $f(t, \cdot, \cdot)$ is concave ( jointly in consumption and utility) for all $t \in[0, T]$, and for some $\hat{c} \in \mathscr{C}$,

$$
E\left[\int_{0}^{T}\left(\max \left\{f_{v}\left(t, \hat{c}_{t}, V_{t}(\hat{c})\right), 0\right\}\right)^{2} d t\right]<\infty
$$

Then condition $\mathrm{C} 2^{\prime}$ is satisfied (with $m$ defined in Eq. (3)).

Lemma 1 implies in particular that $V_{0}$ is concave if $f$ is concave jointly in its consumption and utility arguments, provided that the assumed integrability condition holds for any $\hat{c} \in \mathscr{C}$. This conclusion generalizes Proposition 5 of Duffie and Epstein [12] by allowing $f_{v}$ to be unbounded. Later we will encounter applications in which $V_{0}$ is concave, but $f$ is not jointly concave in consumption and utility.

\subsection{First Order Conditions}

Let the function $I:[0, T] \times \mathbb{R}^{2} \rightarrow \mathbb{R}^{+}$be defined by

$$
\begin{aligned}
I(t, x, v)=0, & \text { if } \quad e^{x} \geqslant f_{c}(t, 0, v) ; \\
f_{c}(t, I(t, x, v), v)=e^{x}, & \text { if } \quad e^{x}<f_{c}(t, 0, v) .
\end{aligned}
$$


Assuming that $V_{0}$ is strictly increasing (a consequence of $\mathrm{C} 2$ and $\mathrm{C} 4$ ), the first-order conditions under SDU are equivalent to the system

$$
\begin{aligned}
X_{t}= & \log (\lambda)-\int_{0}^{t}\left(f_{v}\left(s, I\left(s, X_{s}, V_{s}\right), V_{s}\right)+r_{s}+\frac{1}{2} \eta_{s}^{\prime} \eta_{s}\right) d s \\
& -\int_{0}^{t} \eta_{s}^{\prime} d B_{s}, \\
V_{t}=E_{t}\left[\int_{t}^{T} f\left(s, I\left(s, X_{s}, V_{s}\right), V_{s}\right) d s\right], & \\
& E\left[\int_{0}^{T} \pi_{t} I\left(t, X_{t}, V_{t}\right) d t\right]=w, \quad \lambda \in \mathbb{R}^{++} .
\end{aligned}
$$

Proposition 2. Given $\mathrm{C} 1, \mathrm{C} 2, \mathrm{C} 3$, and $\mathrm{C} 4$, every optimal consumption process $c \in \mathscr{C}$ takes the form $c_{t}=I\left(t, X_{t}, V_{t}\right), t \in[0, T]$, where $(X, V, \lambda) \in$ $D \times \mathscr{V} \times \mathbb{R}^{++}$is a solution to (4). Conversely, if conditions $\mathrm{C} 1, \mathrm{C} 2^{\prime}, \mathrm{C} 3$, and $\mathrm{C} 4$ hold for a given $\hat{c} \in \mathscr{C}$ of this form, then $\hat{c}$ is optimal.

Proof. Defining $X_{t}(\lambda, c)=\log \left(\lambda \pi_{t}\right)-\int_{0}^{t} f_{v}\left(s, c_{s}, V_{s}(c)\right) d s$, the first-order conditions, under our assumptions, can be written as $c_{t}=I\left(t, X_{t}(\lambda, c), V_{t}(c)\right)$ and $\langle\pi, c\rangle=w$. The result is an easy consequence of this observation and Proposition 1.

The system of equations (4a), (4b) is a forward-backward stochastic differential equation (FBSDE) system, $X$ being the forward component, and $V$ being the backward component. General existence-uniqueness results for this type of equations that do not rely on additional Markovian structure and PDE techniques are lacking. Antonelli [1] presents some related results under conditions that are likely to be violated in this context. Antonelli's examples illustrate what can go wrong if his assumptions are relaxed. In our context, Proposition 1(c) shows that a solution to (4) is guaranteed if $V_{0}$ is continuous and concave, and $\mathscr{C}$ is closed and convex.

\subsection{Computational Approach}

Computationally, system (4) can be approached by the methodology of Ma et al. [39], as we now show. A Markovian structure is required for this purpose. We therefore assume that the following condition holds throughout this subsection: 
C5. (a) The functions $\mu^{Y}:[0, T] \times \mathbb{R}^{m} \rightarrow \mathbb{R}^{m}$, and $\sigma^{Y}:[0, T] \times$ $\mathbb{R}^{m} \rightarrow \mathbb{R}^{n}$ are such that the following SDE has a unique (strong) solution, $Y$, valued in $\mathbb{R}^{m}$ :

$$
Y_{t}=Y_{0}+\int_{0}^{t} \mu^{Y}\left(s, Y_{s}\right) d s+\int_{0}^{t} \sigma^{Y}\left(s, Y_{s}\right) d B_{s}
$$

(b) For some (measurable) functions $r:[0, T] \times \mathbb{R}^{m} \rightarrow \mathbb{R}$ and $\eta:[0, T] \times \mathbb{R}^{m} \rightarrow \mathbb{R}^{n}, r_{t}=r\left(t, Y_{t}\right)$ and $\eta_{t}=\eta\left(t, Y_{t}\right)$.

In part (b) we use $r$ and $\eta$ to represent both the stochastic processes and the corresponding functions of the underlying Markov state. The meaning will always be clear from the context. Restrictions on $\mu^{Y}$ and $\sigma^{Y}$ that guarantee C5(a) are well known and can be found, for example, in Karatzas and Shreve [32]. In the case in which $r$ and $\eta$ are deterministic processes, the process $Y$ should be taken to be the empty function.

The key idea is that in a solution of (4), $V_{t}$ should be a function of $\left(X_{t}, Y_{t}\right)$, for every $t$. So let us hypothesize the existence of a function $g:[0, T] \times \mathbb{R}^{m+1} \rightarrow \mathbb{R}$ such that $V_{t}=g\left(t, X_{t}, Y_{t}\right)$ for all $t$. The dynamics of $X$ become

$$
X_{t}=\log (\lambda)+\int_{0}^{t} \rho\left(s, X_{s}, Y_{s}, g\left(s, X_{s}, Y_{s}\right)\right) d s-\int_{0}^{t} \eta\left(s, Y_{s}\right)^{\prime} d B_{s},
$$

where the function $\rho$ is defined by

$$
\rho(t, x, y, v)=-f_{v}(t, I(t, x, v), v)-r(t, y)-\frac{1}{2} \eta(t, y)^{\prime} \eta(t, y) .
$$

Applying Ito's lemma, we are led naturally to the following quasilinear PDE for $g$ (where the arguments of $g$ and its derivatives are omitted):

$$
\begin{aligned}
-f(t, I(t, x, g), g)= & g_{x} \rho(t, x, y, g)+g_{y} \mu^{Y}(t, y)+g_{t}-g_{x y}^{\prime} \sigma^{Y}(t, y) \eta(t, y) \\
& +\frac{1}{2} g_{x x} \eta(t, y)^{\prime} \eta(t, y) \\
& +\frac{1}{2} \operatorname{tr}\left(g_{y y} \sigma^{Y}(t, y) \sigma^{Y}(t, y)^{\prime}\right), \\
g(T, \cdot, \cdot)= & 0 .
\end{aligned}
$$

Notice that the above PDE does not involve the Lagrange multiplier $\lambda$.

Proposition 3. Suppose that C5 holds, g solves PDE (6), and

$$
E\left[\left(\int_{0}^{T}\left\|g_{x}\left(t, X_{t}, Y_{t}\right) \eta_{t}^{\prime}\left(t, Y_{t}\right)-g_{y}\left(t, X_{t}, Y_{t}\right) \sigma^{Y}\left(t, Y_{t}\right)\right\|^{2} d t\right)^{1 / 2}\right]<\infty .
$$


For any $\lambda>0$, if $X$ solves $S D E(5)$, and $V_{t}=g\left(t, X_{t}, Y_{t}\right)$, then $(X, V)$ solves the FBSDE system (4a), (4b).

Proof. The result is an immediate consequence of Ito's lemma, condition (7) ensuring that the local martingale part of the expansion is a martingale.

A numerical implementation of this approach to solving the FBSDE system (4a), (4b) can be based on the procedure of Douglas et al. [10].

Propositions 2 and 3 imply that if we can find a function $g$ that solves (6) and satisfies (7), then the agent's problem reduces to the simple task of finding a value for $\lambda$ that makes the budget constraint tight. Given the Markovian specification of optimal consumption, it is a standard exercise to find a corresponding financing trading strategy in short-term borrowing or lending and $m+1$ funds, each one of which is chosen to be instantaneously perfectly correlated with $X$ or a component of $Y$.

The results of Ma et al. [39] show that the following restrictions are sufficient for the existence of a solution to (6) that satisfies (7) (in particular implying that $g_{x}$ and $g_{y}$ are bounded):

(a) There exists $\varepsilon>0$ such that, for all $(t, y) \in[0, T] \times \mathbb{R}^{m}$, the smallest eigenvalue of $\left[\eta(t, y), \sigma^{Y}(t, y)^{\prime}\right]^{\prime}\left[\eta(t, y), \sigma^{Y}(t, y)^{\prime}\right]$ is at least $\varepsilon$.

(b) The functions $\eta(t, y), \mu^{Y}(t, y), \sigma^{Y}(t, y)$, and $f(t, I(t, x, 0), 0)$ are all bounded, and $|\rho(t, x, y, v)| \leqslant \phi(|v|)$ for all $x, y, v$ and $t$, for some function $\phi$.

In the case of deterministic $r$ and $\eta$, the conditions simplify further to (a) $\eta$ is bounded away from zero, and (b) the function $f(t, I(t, x, 0), 0)$ is bounded, and $|\rho(t, x, v)| \leqslant \phi(|v|)$ for all $x, v$, and $t$, for some function $\phi$. All of these conditions are far from necessary for Proposition 3 to apply, however. In fact, in the remainder of this paper we will discuss exclusively problems that violate the above conditions.

\section{A CLASS OF HOMOTHETIC SDU}

This section analyzes a parametric homothetic SDU specification, for which the solution method of the last section simplifies significantly. The main results are existence and basic properties of the utility function and the sufficiency of the first-order conditions for optimality. The section concludes with expressions for optimal consumption plans and portfolios in some simple special cases. The general solution of the agent's problem for this SDU class is the topic of the following section. 


\subsection{Utility Specification}

We begin by restricting the space of consumption processes: ${ }^{6}$

$$
\mathscr{C}=\left\{c \in D^{++}: E\left(\int_{0}^{T} c_{t}^{l} d t\right)<\infty \text { for all } l \in \mathbb{R}\right\} .
$$

The intertemporal aggregator we consider takes one of the forms

$$
f(c, v)= \begin{cases}(1+\alpha)\left(\left(c^{\gamma} / \gamma\right)|v|^{\alpha /(1+\alpha)}-\beta v\right), & \text { if } \gamma \neq 0, \\ (1+\alpha v)[\log (c)-(\beta / \alpha) \log (1+\alpha v)], & \text { if } \gamma=0,\end{cases}
$$

with parameter restrictions: ${ }^{7}$

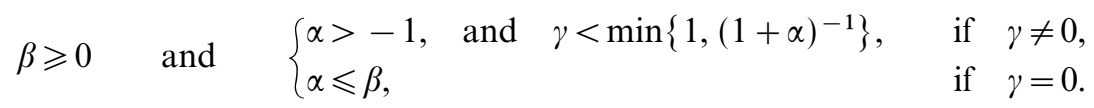

We have omitted the time argument of $f$, since $f$ is time-independent in this formulation. The consumption argument, $c$, is restricted to be strictly positive, and for $\gamma=0$, the utility argument, $v$, is restricted to be greater than $-1 / \alpha$. For $\alpha=\gamma=0$ we interpret ( $8 \mathrm{~b}$ ) by taking a limit, that is, $f(c, v)=\log (c)-\beta v$.

Ordinally equivalent utility processes are defined by

$$
\hat{V}_{t}= \begin{cases}V_{t}^{1 /(1+\alpha)}, & \text { if } \gamma>0 \\ -\left|V_{t}\right|^{1 /(1+\alpha)}, & \text { if } \gamma<0 \\ \alpha^{-1} \log \left(1+\alpha V_{t}\right), & \text { if } \gamma=0\end{cases}
$$

The backward SDE satisfied by this version of the utility takes the more intuitive form (omitting the argument of $\hat{V}=\hat{V}(c)$ )

$$
\hat{V}_{t}= \begin{cases}E_{t}\left[\int_{t}^{T} e^{-\beta(s-t)}\left(\left(c_{s}^{\gamma} / \gamma\right) d s+(\alpha / 2) \hat{V}_{s}^{-1} d[\hat{V}]_{s}\right)\right], & \text { if } \gamma \neq 0, \\ E_{t}\left[\int_{t}^{T} e^{-\beta(s-t)}\left(\log \left(c_{s}\right) d s+(\alpha / 2) d[\hat{V}]_{s}\right)\right], & \text { if } \gamma=0,\end{cases}
$$

where $[\hat{V}]$ denotes the quadratic variation of $\hat{V}$.

\footnotetext{
${ }^{6}$ For simplicity, the integrability restrictions we impose are stronger than necessary for our proofs to go through, but have the advantage that they are independent of utility parameters.

${ }^{7}$ For $\gamma \neq 0$, the restriction $\alpha>-1$ is assumed to keep utility finite near $T$. This issue can be easily finessed by introducing a non-zero bequest function (with arbitrarily small weight), as outlined in Section 7, where we present an interesting example with $\alpha=-1$. For $\alpha<-1$ the utility function to be maximized must be defined as $U(c)=-V_{0}(c)$. Concavity and monotonicity of $U$ (given the second inequality of $(8 \mathrm{c}))$ can then be proved as in Theorem 1 .
} 
The parameter $\alpha$ clearly has no impact on preferences over the set of deterministic consumption paths. It is a measure of comparative risk aversion in a sense defined by Duffie and Epstein [12], as well as a measure of preferences for the timing of resolution of uncertainty in a sense defined by Skiadas [47] (for a related application, see Duffie et al. [19]). If $\gamma>0$, then $\hat{V}_{t}>0$ for all $t<T$, and a negative $\alpha$ penalizes variability of the utility process. Therefore, if $\gamma>0$, risk aversion increases as the value of $\alpha$ decreases, a negative $\alpha$ indicates preferences for early resolution, and a positive $\alpha$ indicates preferences for late resolution. The same conclusions are valid if $\gamma=0$, although $\hat{V}$ need not be uniformly signed in this case. Finally, if $\gamma<0$, the role of the sign of $\alpha$ is reversed, because in this case $\hat{V}_{t}<0$ for all $t<T$. Therefore, with $\gamma<0$, risk aversion increases with $\alpha$, and a positive (negative) $\alpha$ corresponds to preferences for early (late) resolution of uncertainty.

\subsection{Existence and Basic Properties}

The above SDU specification is ordinally equivalent to a parametric class first studied by Duffie and Epstein $[12,13]$, who also explain in what sense the functional form for $\gamma=0$ is a limiting case (under appropriate normalization) of the case for $\gamma \neq 0$. The above SDU can also be obtained as a continuous-time limit of the CES Kreps-Porteus utility specification used by Epstein and Zin [24]. Nevertheless, a general proof of existence and basic properties has been lacking. The aggregator, $f$, defined in (8) violates the usual Lipschitz and growth conditions used by Duffie and Epstein [12] and Pardoux and Peng [43] to prove existence and uniqueness of the backward SDE underlying the SDU definition. Duffie and Lions [16] give a PDE characterization of the utility process (for the infinite horizon case), but their Markovian restrictions on consumption plans are not appropriate in the current setting, and their parameter restrictions rule out interesting parameter ranges satisfying (8c). Instead, we will base our proof of existence and basic properties on our probabilistic results on backward SDEs of Appendix A.

To state the main existence result, we introduce the space

$$
D_{0}=\left\{V \in D: E\left[\operatorname{ess} \sup _{t}\left|V_{t}\right|^{l}\right]<\infty \text {, for every } l>0\right\},
$$

and its strictly positive cone $D_{0}^{++}=\left\{V \in D_{0}: V>0\right.$ almost everywhere $\}$. Throughout our discussion of the utility specification (8), we will assume that the utility process set $\mathscr{V}$ is given as

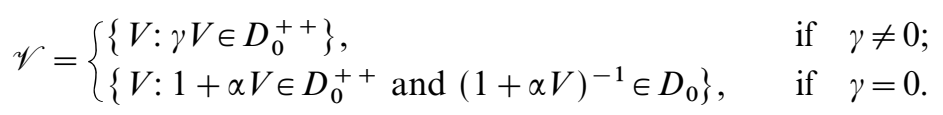


THEOREM 1. Suppose that $\mathscr{C}$ and $f$ are specified by (8). Then recursion (2) is satisfied by a unique $V(c) \in \mathscr{V}$. Moreover, $V_{0}$ is strictly concave, increasing, and homothetic. ${ }^{8}$

Other interesting properties of $V_{0}$ involve comparative risk aversion and preferences for the timing of resolution of uncertainty, as briefly discussed above. The respective formal treatments of Duffie and Epstein [12, Proposition 6] and Skiadas [47, Appendix A] are based on Lipschitz conditions that are violated here. Given our results in Appendix A, however, adapting their arguments to the present setting becomes a tractable exercise that we leave to the interested reader.

A relevant utility property that Theorem 1 does not cover is the existence and form of the utility gradient. (Once again, the utility gradient computations of Duffie and Skiadas [20] do not apply in this context.) Due to integrability issues, condition C2 may not be satisfied for all parameter values specified in $(8 \mathrm{c})$. However, since the unique optimal consumption plan will later be specified in closed form, all that we need is the verification of condition $\mathrm{C}^{\prime}$ at the optimum, which is accommodated by the following result.

Lemma 2. Suppose that $\mathscr{C}$ and $f$ are specified by (8). For condition $\mathrm{C} 2$ ' to hold with $m$ given by Eq. (3), it is sufficient that either $\alpha \gamma=0$, or $\alpha \gamma \neq 0$ and $E\left[\exp \left(3 \int_{0}^{T-\varepsilon}\left|f_{v}\left(\hat{c}_{t}, V_{t}(\hat{c})\right)\right|\right) d t\right]<\infty$ for every $\varepsilon \in(0, T)$.

The integrability condition of Lemma 2 is required only when both $\alpha \neq 0$ and $\gamma \neq 0$, and even then it can be weakened, as indicated in the proof of Lemma 2. Nevertheless, the above form of the lemma will suffice for optimality verification in our applications below.

\subsection{Optimal Consumption and Portfolios for Some Special Cases}

Significant simplifications to the solution of the agent's problem result if one or more of the following conditions are satisfied:

I. Time-additive utility: $\alpha=0$.

II. Logarithmic SDU: $\gamma=0$.

III. Deterministic investment opportunity set: $r$ and $\eta$ are deterministic.

For easy reference, we summarize these simplifications in the following theorem, making use of two auxiliary processes,

$$
k_{t}= \begin{cases}\beta\left[\beta-\alpha\left(1-e^{-\beta(T-t)}\right)\right]^{-1}, & \text { if } \gamma=0 \\ (1-\gamma(1+\alpha))^{-1}, & \text { if } \gamma \neq 0\end{cases}
$$

${ }^{8} V_{0}$ is homothetic if, for all $k>0, V_{0}(c) \geqslant V_{0}\left(c^{\prime}\right) \Leftrightarrow V_{0}(k c) \geqslant V_{0}\left(k c^{\prime}\right)$. 
and

$$
q_{t}=\frac{\beta}{1-\gamma}-\frac{\gamma}{1-\gamma}\left(r_{t}+\frac{k_{t}}{2} \eta_{t} \cdot \eta_{t}\right)
$$

The form of the solution below under a deterministic investment opportunity set has been previously derived by Svensson [49] and Obstfeld [42] (who, however, did not prove optimality or that the utility is well defined.) Here, and in the following section, we will make the assumption that $r$ and $\eta$ are bounded in order to avoid long technical discussions relating to integrability issues. Depending on parameters, several of our proofs apply with weaker restrictions on $r$ and $\eta$.

THeOREm 2. Suppose that $\mathscr{C}$ and $f$ are specified by (8), and $r$ and $\eta$ are bounded.

(a) Suppose that at least one of conditions I and III holds. Then the optimal consumption plan follows the dynamics

$$
\frac{d c_{t}}{c_{t}}=\left(r_{t}-q_{t}+k_{t} \eta_{t} \cdot \eta_{t}\right) d t+k_{t} \eta_{t}^{\prime} d B_{t} .
$$

(b) Suppose that at least one of conditions II and III holds. Then the optimal consumption to wealth ratio is

$$
\frac{c_{t}}{W_{t}(c)}=\left[\int_{t}^{T} \exp \left(-\int_{t}^{s} q_{\tau} d \tau\right) d s\right]^{-1} .
$$

(c) Suppose that either condition III holds, or conditions I and II hold (that is, $\alpha=\gamma=0$ ), or all three conditions hold. Then the optimal trading strategy is

$$
\psi_{t}=k_{t}\left(\sigma_{t}^{R} \sigma_{t}^{R^{\prime}}\right)^{-1} \mu_{t}^{R} .
$$

Under the assumptions of part (c) of the theorem, the optimal portfolio is instantaneously mean-variance efficient, even without additivity. But, compared to the Merton solution $(\alpha=0)$, less is invested in the risky fund if there are preferences for early resolution of uncertainty $(\gamma \alpha<0$, or $\gamma=0$ and $\alpha<0$ ), and more is invested in the risky fund if there are preferences for late resolution $(\gamma \alpha>0$, or $\gamma=0$ and $\alpha>0)$. An interesting observation is that if $\gamma=0$, and $r$ and $\eta$ are deterministic and constant over time, then, where Merton's solution involves constant over time portfolio weights, for $\alpha \neq 0$, the optimal positions change deterministically over time, approaching the Merton solution toward the end of the planning horizon, $T$. 
An example with non-additive SDU in which the optimal portfolio is instantaneously mean-variance efficient under any state price dynamics is outlined in Section 7.

\section{GENERAL SOLUTION METHOD FOR HOMOTHETIC SDU CLASS}

This section presents a general solution method for the SDU specification of Theorem 1 and a stochastic investment opportunity set.

\subsection{A Convenient Change of Measure}

In discussing cases not covered by Theorem 2 , it will be convenient to express certain backward SDEs in terms of a new probability, $\widetilde{P}$, defined as follows. Recalling that $k$ is given by (11), we define the new probability $\widetilde{P}$ through its density:

$$
E_{t}\left[\frac{d \tilde{P}}{d P}\right] \equiv \xi_{t} \equiv \exp \left(-\frac{1}{2} \int_{0}^{t}\left(1-k_{s}\right)^{2} \eta_{s} \cdot \eta_{s} d s-\int_{0}^{t}\left(1-k_{s}\right) \eta_{s}^{\prime} d B_{s}\right) .
$$

(Since $r$ and $\eta$ are assumed bounded, the right-hand side defines a martingale and $(12 a)$ is therefore consistent.) The expectation operator with respect to $\widetilde{P}$ will be denoted $\widetilde{E}$, and the corresponding conditional expectation given $\mathscr{F}_{t}$ will be denoted $\widetilde{E}_{t}$. In particular, for any random variable $Z$, $\widetilde{E}_{t}[Z]=E_{t}\left[\xi_{T} Z\right] / \xi_{t}$. By Girsanov's theorem, the process $\widetilde{B}$, defined by

$$
\widetilde{B}_{t}=B_{t}+\int_{0}^{t}\left(1-k_{s}\right) \eta_{s} d s
$$

is $n$-dimensional standard Brownian motion under the measure $\widetilde{P}$.

\subsection{The Case of Zero Gamma}

Throughout this subsection, we assume that $\mathscr{C}$ and $f$ are specified by (8) with $\gamma=0$, and therefore $k_{t}=\beta\left[\beta-\alpha\left(1-e^{-\beta(T-t)}\right)\right]^{-1}$.

In order to solve the first-order conditions, we introduce the auxiliary processes $(J, Z) \in D_{0}^{\exp } \times D^{n}$ (where $D_{0}^{\exp }$ is defined in Appendix A) as the unique adapted solution to the backward SDE

$$
\begin{aligned}
d J_{t}= & -\left[\left(1-k_{t}\right)\left(\beta-r_{t}-\frac{k_{t}}{2} \eta_{t} \cdot \eta_{t}\right)+k_{t}(\alpha-\beta) J_{t}+\frac{1}{2} Z_{t} \cdot Z_{t}\right] d t \\
& +Z_{t} d \tilde{B}_{t}, \\
J_{T}= & 0 .
\end{aligned}
$$


Existence and uniqueness of a solution are guaranteed by Theorem A1 of Appendix A. Simple solutions are obtained if $\alpha=\beta$ or if $\alpha=0$. Lemma A1 implies that

$$
\exp \left(J_{t}\right)=\tilde{E}_{t}\left[\exp \left(\int_{t}^{T}\left(1-k_{s}\right)\left(\beta-r_{s}-\frac{k_{s}}{2} \eta_{s} \cdot \eta_{s} d s\right)\right)\right], \quad \text { if } \quad \alpha=\beta .
$$

For $\alpha=0$, the unique solution is the zero solution. The PDE characterization of (13) in a Markovian setting is discussed at the end of this section.

Lemma 3. Suppose that $\mathscr{C}$ and $f$ are defined by (8) with $\gamma=0$, and $r$ and $\eta$ are bounded. Then, given any $\lambda>0$, the FBSDE system (4a), (4b) has a unique solution $(X, V)$ in $D \times \mathscr{V}$. The process $X$ is given by

$$
d X_{t}=-\left[\left((\beta-\alpha) k_{t}-\beta\right) X_{t}+(\alpha-\beta) J_{t}-\beta+r_{t}+\frac{\eta_{t} \cdot \eta_{t}}{2}\right] d t-\eta_{t}^{\prime} d B_{t},
$$

with initial value $X_{0}=\log (\lambda)$, while $V$ satisfies

$$
1+\alpha V_{t}=\exp \left(J_{t}+\left(1-k_{t}\right) X_{t}\right) .
$$

In terms of the solution $(X, V)$ of Lemma 3, the optimal consumption plan is given by

$$
c_{t}=I\left(t, X_{t}, V_{t}\right)=e^{-X_{t}\left(1+\alpha V_{t}\right)}=\exp \left(J_{t}-k_{t} X_{t}\right) .
$$

To complete the solution, we need to determine the value of $\lambda$. Part (b) of Theorem 2 implies that the optimal consumption-to-wealth ratio is

$$
\frac{c_{t}}{W_{t}(c)}=\frac{\beta}{1-e^{-\beta(T-t)}} .
$$

In particular, $c_{0}=\beta w /\left(1-e^{-\beta T}\right)$, which together with (15) determines the value of $\lambda$ (and hence the initial value of $X$ ). Applying Ito's lemma to (15) gives the dynamics of the optimal consumption plan (without having to solve for $\lambda$ first):

$$
\frac{d c_{t}}{c_{t}}=\mu_{t}^{c} d t+\sigma_{t}^{c} d B_{t}
$$

THeOREM 3. Suppose that $\mathscr{C}$ and $f$ are defined by (8) with $\gamma=0$, and $r$ and $\eta$ are bounded. Then the dynamics of the (unique) optimal consumption plan are given by (17), where

$$
\mu_{t}^{c}=r_{t}-\beta+\sigma_{t}^{c} \cdot \eta_{t} \quad \text { and } \quad \sigma_{t}^{c}=k_{t} \eta_{t}^{\prime}+Z_{t} .
$$


The optimal consumption rate as a fraction of wealth is given by (16), and the corresponding optimal trading strategy is

$$
\psi_{t}=k_{t}\left(\sigma_{t}^{R} \sigma_{t}^{R^{\prime}}\right)^{-1} \mu_{t}^{R}+\left(\sigma_{t}^{R^{\prime}}\right)^{-1} Z_{t} .
$$

The first term of the optimal trading strategy is the instantaneously mean-variance efficient strategy of Theorem 2(c). The second term represents the deviation from mean-variance efficiency due to the presence of both a stochastic investment opportunity set, and time non-additivity.

\subsection{The Case of Non-zero Gamma}

Throughout this Section, we assume that $\mathscr{C}$ and $f$ are given by (8) with $\gamma \neq 0$, and hence $k_{t}=k \equiv(1-\gamma(1+\alpha))^{-1}$. The analysis is analogous to the zero gamma case, except that now the optimal consumption to wealth ratio is typically stochastic.

Applying Theorem A2 (of Appendix A), we uniquely define the (progressively measurable) processes $(J, Z)$, where $\gamma J \in D_{0}^{++}$and $\int_{0}^{T} Z_{t}$. $Z_{t} d t<\infty$ a.s., as the solution to the backward SDE:

$$
\begin{aligned}
d J_{t}= & -\left[\frac{1}{\gamma}(1+\alpha)^{\gamma /(1-\gamma)}+\frac{\gamma}{1-\gamma}\left(r_{t}-\frac{\beta}{\gamma}+\frac{k}{2} \eta_{t} \cdot \eta_{t}\right) J_{t}+\frac{\alpha k}{2} \frac{Z_{t} \cdot Z_{t}}{J_{t}}\right] d t \\
& +Z_{t} d \widetilde{B}_{t}, \quad t<T \\
J_{T}= & J_{T-}=0 .
\end{aligned}
$$

In the additive case, $J$ is given by

$$
\begin{gathered}
J_{t}=\frac{1}{\gamma} \tilde{E}_{t}\left[\int_{t}^{T} \exp \left(\frac{\gamma}{1-\gamma} \int_{t}^{s}\left(r_{u}-\frac{\beta}{\gamma}+\frac{k}{2} \eta_{u} \cdot \eta_{u}\right) d u\right) d s\right], \\
\text { if } \quad \alpha=0 .
\end{gathered}
$$

The PDE characterization of (18) in a Markovian setting is discussed at the end of this section.

Lemma 4. Suppose that $\mathscr{C}$ and $f$ are defined by (8) with $\gamma \neq 0$, and $r$ and $\eta$ are bounded. Then, given any $\lambda>0$, the FBSDE system (4a), (4b) has a unique solution $(X, V)$ in $D \times \mathscr{V}$. The process $X$ is given by

$$
d X_{t}=-\left[\frac{\alpha}{\gamma}(1+\alpha)^{\gamma /(1-\gamma)} J_{t}^{-1}-(1+\alpha) \beta+r_{t}+\frac{\eta_{t} \cdot \eta_{t}}{2}\right] d t-\eta_{t}^{\prime} d B_{t},
$$

with initial value $X_{0}=\log (\lambda)$, while $V$ satisfies

$$
V_{t}=(\gamma /|\gamma|)\left|J_{t}\right|^{1+\alpha k} \exp \left((1-k) X_{t}\right)
$$


In terms of $(X, V)$, the optimal consumption plan is

$$
\begin{aligned}
c_{t} & =I\left(t, X_{t}, V_{t}\right)=(1+\alpha)^{1 /(1-\gamma)} \exp \left(-\frac{X_{t}}{1-\gamma}\right)\left|V_{t}\right|^{\alpha k / 1+\alpha k} \\
& =(1+\alpha)^{1 /(1-\gamma)} \exp \left(-k X_{t}\right)\left|J_{t}\right|^{\alpha k} .
\end{aligned}
$$

Ito's lemma then delivers the optimal consumption dynamics.

THEOREM 4. Suppose that $\mathscr{C}$ and $f$ are defined by (8) with $\gamma \neq 0$, and $r$ and $\eta$ are bounded. Then the dynamics of the optimal consumption plan are given by (17), where

$$
\begin{aligned}
& \mu_{t}^{c}=\frac{1}{1-\gamma}\left(r_{t}-\beta\right)+\sigma_{t}^{c} \cdot \eta_{t}+\frac{k}{2}\left(\frac{\gamma}{1-\gamma} \eta_{t} \cdot \eta_{t}-\frac{\alpha}{J_{t}^{2}} Z_{t} \cdot Z_{t}\right), \\
& \sigma_{t}^{c}=k\left(\eta_{t}^{\prime}+\frac{\alpha}{J_{t}} Z_{t}\right) .
\end{aligned}
$$

The optimal consumption-to-wealth ratio is given by

$$
\frac{c_{t}}{W_{t}(c)}=\gamma^{-1}(1+\alpha)^{\gamma /(1-\gamma)} J_{t}^{-1}
$$

The optimal trading strategy is

$$
\psi_{t}=k\left(\sigma_{t}^{R} \sigma_{t}^{R^{\prime}}\right)^{-1} \mu_{t}^{R}+(1+\alpha k)\left(\sigma_{t}^{R^{\prime}}\right)^{-1} \frac{Z_{t}}{J_{t}}
$$

As in the zero gamma case, the first term of the optimal portfolio expression represents an instantaneously mean-variance efficient allocation, while the second term represents deviations from mean-variance efficiency due to a stochastic investment opportunity set. Formally setting $\alpha=-1$ in the above expression results in an instantaneously mean-variance efficient portfolio, for any price dynamics. This limiting case (not covered by Theorem 4) is revisited in Section 7.

\subsection{PDE Characterization of $(J, Z)$}

Given the above analysis, the agent's problem reduces to the computationally well understood problem of solving the backward SDE that defines $(J, Z)$. Below, we outline the PDE characterization of such a solution in a Markovian setting. 
In the remainder of this Section, we assume the Markovian structure of condition $\mathrm{C} 5$. We also recall the change of measure (12), and we use the dynamics of $Y$ in the form $d Y_{t}=\tilde{\mu}^{Y}\left(t, Y_{t}\right) d t+\sigma^{Y}\left(t, Y_{t}\right) d \tilde{B}_{t}$, where

$$
\tilde{\mu}^{Y}(t, y)=\mu^{Y}(t, y)-\left(1-k_{t}\right) \sigma^{Y}(t, y) \eta(t, y) .
$$

Letting $J_{t}=h\left(t, Y_{t}\right)$ for some function $h$, and using Ito's lemma, the BSDE characterizing $(J, Z)$ leads naturally to a PDE, stated below for various parameter ranges. In all cases, the stated PDE can be viewed as a simplified version of $(6)$ for the given parametric SDU class, obtained by letting

$$
g(t, x, y)= \begin{cases}\alpha^{-1}\left[\exp \left(\left(1-k_{t}\right) x+h(t, y)\right)-1\right], & \text { if } \gamma=0 ; \\ (\gamma /|\gamma|)|h(t, y)|^{1+\alpha k} \exp ((1-k) x), & \text { if } \gamma \neq 0 .\end{cases}
$$

Given a solution, $h$, to the appropriate PDE, the BSDE solution is

$$
J_{t}=h\left(t, Y_{t}\right) \quad \text { and } \quad Z_{t}=h_{y}\left(t, Y_{t}\right) \sigma^{Y}\left(t, Y_{t}\right) .
$$

(In applications, one must of course confirm that $(J, Z)$ are sufficiently integrable.)

We now state the PDE that has to be solved for all parameter ranges for which Theorem 2 does not provide a complete solution for general price dynamics: ${ }^{9}$

Case $1(\gamma=0)$.

$$
\begin{aligned}
(\beta-\alpha) k_{t} h= & \left(1-k_{t}\right)\left(\beta-r-\frac{1}{2} k_{t} \eta^{\prime} \eta\right)+h_{t}+h_{y} \tilde{\mu}^{Y} \\
& +\frac{1}{2} \operatorname{tr}\left[\left(h_{y y}+h_{y}^{\prime} h_{y}\right) \sigma^{Y}\left(\sigma^{Y}\right)^{\prime}\right], \quad h(T, \cdot)=0 .
\end{aligned}
$$

For $\alpha=\gamma=0$, this PDE is also valid, but its solution is zero, corresponding to the optimal portfolio allocation of Theorem 2(c). Another simplification arises if $\alpha=\beta$, as we discussed in Section 5.2.

Case $2(\gamma \neq 0$ and $\alpha \neq 0)$.

$$
\begin{aligned}
-\frac{\gamma}{1-\gamma}\left(r-\frac{\beta}{\gamma}+\frac{k}{2} \eta \cdot \eta\right) h= & \frac{1}{\gamma}(1+\alpha)^{\gamma /(1-\gamma)}+h_{t}+h_{y} \tilde{\mu}^{Y} \\
& +\frac{1}{2} \operatorname{tr}\left[\left(h_{y y}+\frac{\alpha k}{h} h_{y}^{\prime} h_{y}\right) \sigma^{Y}\left(\sigma^{Y}\right)^{\prime}\right], \\
h(T, \cdot)= & 0 .
\end{aligned}
$$

${ }^{9}$ The notation $\operatorname{tr}(A)$, where $A$ is any square matrix, stands for the trace of $A$, that is, the sum of its diagonal elements. For any matrices $A, B$ such that the products $A B$ and $B A$ are well defined, the identity $\operatorname{tr}(A B)=\operatorname{tr}(B A)$ holds. Thus $\operatorname{tr}\left[h_{y}^{\prime} h_{y} \sigma^{Y}\left(\sigma^{Y}\right)^{\prime}\right]=h_{y} \sigma^{Y} \sigma^{Y^{\prime}} h_{y}^{\prime}$ and $\operatorname{tr}\left[h_{y y} \sigma^{Y}\left(\sigma^{Y}\right)^{\prime}\right]=\operatorname{tr}\left[\left(\sigma^{Y}\right)^{\prime} h_{y y} \sigma^{Y}\right]$. 
Case $3(\gamma \neq 0$ and $\alpha=0)$.

In this case, PDE (23) applies, but it can be further simplified. Let us assume that the functions $r(t, y)$ and $\eta(t, y)$ are time independent, allowing us to simplify notation to $r_{t}=r\left(Y_{t}\right)$ and $\eta_{t}=\eta\left(Y_{t}\right)$. (This is no less general than condition $\mathrm{C} 5$, since a time component can be added to $Y$.) Using Eq. (19), we have

$$
h\left(t, Y_{t}\right)=\frac{1}{\gamma} \int_{t}^{T} e^{p\left(s-t, Y_{t}\right)} d s
$$

where

$$
e^{p\left(s-t, Y_{t}\right)}=\tilde{E}_{t} \exp \left[\frac{\gamma}{1-\gamma} \int_{t}^{s}\left(r_{u}-\frac{\beta}{\gamma}+\frac{k}{2} \eta_{u} \cdot \eta_{u}\right) d u\right]
$$

The function $p(\tau, y)$ can be computed as the solution to the PDE

$$
\begin{aligned}
& -\frac{\gamma}{1-\gamma}\left(r-\frac{\beta}{\gamma}+\frac{k}{2} \eta \cdot \eta\right) \\
& \quad=-p_{\tau}+p_{y} \tilde{\mu}^{Y}+\frac{1}{2} \operatorname{tr}\left[\left(p_{y y}+p_{y}^{\prime} p_{y}\right) \sigma^{Y}\left(\sigma^{Y}\right)^{\prime}\right], \quad p(0, \cdot)=0 .
\end{aligned}
$$

\section{EXAMPLES WITH “AFFINE” DYNAMICS}

For the homothetic SDU class of Section 4, we have seen in the last section that the agent's problem reduces to the solution of a single backward SDE, which in general can be solved numerically, for example, by numerically solving an associated quasilinear PDE. The complexity of the last step is a function of the price dynamics specification. For example, a trivial solution results if $r$ and $\eta$ are deterministic. In this section we outline a more general class of price dynamics, familiar from the term-structure literature (see, for example, Duffie and Kan [15]), for which relatively simple solutions to the first-order conditions result.

This section's models are not strictly special cases of Theorems 3 and 4, because $r$ and $\eta$ are not bounded. For some parameter ranges, our exact proofs apply. For other parameter values, however, the utility process can diverge to infinity in finite time, and our verification arguments do not apply, because of integrability issues. Below, we provide some coarse sufficient conditions that keep utility finite, while in some additive cases a complete closed-form solution is possible (and optimality verification is straightforward). A complete characterization, however, requires an 
understanding of the behavior of the underlying affine dynamics that, to our knowledge, is not currently available in the literature and is beyond the scope of this paper.

Throughout the section, we assume the SDU specification of Section 4 and that either $\gamma=0$ or $\alpha=0$. Under the price dynamics described below, we show that the relevant backward SDE reduces to a Riccati equation that can either be solved in closed form or is straightforward to solve numerically.

\subsection{Price Dynamics}

Given any $v \in \mathbb{R}^{n}, \operatorname{diag}(v)$ denotes the diagonal matrix with $v$ as its diagonal, $\sqrt{v}$ denotes the (column) vector $\left(\sqrt{v_{1}}, \ldots, \sqrt{v_{n}}\right)^{\prime}$, and $v^{2}$ denotes $\left(v_{1}^{2}, \ldots, v_{n}^{2}\right)^{\prime}$. Also, given any square matrix $A, A_{i i}$ denotes its $i$ th diagonal entry.

We postulate a state process $Y$, valued in $\mathbb{R}^{n}$, with dynamics

$$
d Y_{t}=\left(\theta-\kappa Y_{t}\right) d t+\Sigma \operatorname{diag}\left(\sqrt{v+\zeta Y_{t}}\right) d B_{t},
$$

where $\theta, v \in \mathbb{R}^{n}$ and $\zeta, \kappa, \Sigma \in \mathbb{R}^{n \times n}$. $Y$ is assumed to be the unique process that is valued in

$$
\mathscr{Y}=\left\{y \in \mathbb{R}^{n}: v_{i}+\zeta_{i} y>0, \text { for all } i\right\},
$$

and satisfies (25). We refer to Duffie and Kan [15] and Dai et al. [9] for conditions on the parameters sufficient for this assumption to hold.

The price dynamics we consider are of the following two types:

Model A. $r_{t}=a+b \cdot Y_{t}$ and $\eta_{t}=\operatorname{diag}\left(\sqrt{v+\zeta Y_{t}}\right) \Phi$, where $a \in \mathbb{R}$ and $b, \Phi \in \mathbb{R}^{n}$.

Model B. $\quad r_{t}=a_{r}+b_{r}^{\prime} Y_{t}+Y_{t}^{\prime} c_{r} Y_{t}$ and $\eta_{t}=a_{\eta}+b_{\eta} Y_{t}$, where $a_{r} \in \mathbb{R}$, $a_{\eta}, b_{r} \in \mathbb{R}^{n}$, and $b_{\eta}, c_{r} \in \mathbb{R}^{n \times n}$, and $c_{r}$ is symmetric. Moreover, $\zeta=0$ and $v_{i}=1$ for all $i$.

Given the above assumption, we show below how to solve the appropriate PDE of Section 5.4. The proposed solution to the consumptionportfolio problem is then formally constructed as in Theorems 3 and 4 .

\subsection{The Case of Zero Gamma}

In this Section, we assume that $f$ is given by (8) with $\gamma=0$.

For Model A, we conjecture that PDE (22) has an affine solution,

$$
h(t, y)=G_{t}+H_{t} \cdot y,
$$

where $H$ and $G$ are functions of time valued in $\mathbb{R}^{n}$ and $\mathbb{R}$, respectively. 
Direct computation shows that $h$ solves (22) if $G, H$ solve

$$
\begin{aligned}
\dot{H}_{t}= & {\left[(\beta-\alpha) k_{t} I+\kappa^{\prime}+\left(1-k_{t}\right) \zeta^{\prime} \operatorname{diag}(\Phi) \Sigma^{\prime}\right] H_{t}+\left(1-k_{t}\right)\left[b+\frac{k_{t}}{2} \zeta^{\prime} \Phi^{2}\right] } \\
& -\frac{1}{2} \zeta^{\prime}\left(\Sigma^{\prime} H_{t}\right)^{2}, \quad H_{T}=0, \\
\dot{G}_{t}= & (\beta-\alpha) k_{t} G_{t}-\left(1-k_{t}\right)\left[\beta-a-\frac{k_{t}}{2} v^{\prime} \Phi^{2}\right] \\
& -H_{t}^{\prime}\left[\theta-\left(1-k_{t}\right) \Sigma \operatorname{diag}(v) \Phi+\frac{1}{2} \Sigma \operatorname{diag}(v) \Sigma^{\prime} H_{t}\right], \quad G_{T}=0 .
\end{aligned}
$$

The first equation is a Riccati equation in $H$, and although straightforward to solve numerically, it can diverge to infinity in finite time. A sufficient condition that precludes this is given by the following result:

Lemma 5. Suppose that $b, \zeta \geqslant 0$, and $\sum_{i} \zeta_{i j}>0$ for all $j$. If $\alpha<0$, then the Riccati equation in $H$ has a finite solution for any finite $T$.

Given $H, G$ is easily computed using the second equation. A closed form solution can easily be obtained in the infinite horizon version of this model $(T=\infty)$, if, for example, $\zeta, \kappa$, and $\Sigma$ are diagonal.

For model B, direct computation shows that a solution to PDE (22) is given by

$$
h(t, y)=C_{t}+D_{t}^{\prime} y+y^{\prime} F_{t} y,
$$

with $F$ symmetric, and where $C, D$, and $F$ are deterministic functions of time, obtained by solving the following three equations, in order: ${ }^{10}$

$$
\begin{aligned}
\dot{F}_{t}= & \left(1-k_{t}\right)\left(c_{r}+\frac{k_{t}}{2} b_{\eta}^{\prime} b_{\eta}\right)+(\beta-\alpha) k_{t} F_{t}+\left[\kappa+\left(1-k_{t}\right) \Sigma b_{\eta}\right]^{\prime} F_{t} \\
& +F_{t}\left[\kappa+\left(1-k_{t}\right) \Sigma b_{\eta}\right]-2 F_{t} \Sigma \Sigma^{\prime} F_{t}, \quad F_{T}=0 . \\
\dot{D}_{t}= & {\left[(\beta-\alpha) k_{t} I+\kappa^{\prime}-2 F_{t}^{\prime} \Sigma \Sigma^{\prime}+\left(1-k_{t}\right) b_{\eta}^{\prime} \Sigma^{\prime}\right] D_{t}+\left(1-k_{t}\right)\left[b_{r}+k_{t} b_{\eta}^{\prime} a_{\eta}\right] } \\
& -2 F_{t}\left[\theta-\left(1-k_{t}\right) \Sigma a_{\eta}\right], \quad D_{T}=0, \\
\dot{C}_{t}= & k_{t}(\beta-\alpha) C_{t}-\left(1-k_{t}\right)\left[\beta-a_{r}-\frac{k_{t}}{2} a_{\eta}^{\prime} a_{\eta}\right] \\
& -D_{t}^{\prime}\left[\theta-\left(1-k_{t}\right) \Sigma a_{\eta}+\frac{1}{2} \Sigma \Sigma^{\prime} D_{t}\right]-\operatorname{tr}\left(F_{t} \Sigma \Sigma^{\prime}\right), \quad C_{T}=0 .
\end{aligned}
$$

${ }^{10}$ We use the fact that $y^{\prime} A y=0, \forall y$, for some $A \in \mathbb{R}^{n \times n}$ if and only if $A+A^{\prime}=0$. 
Lemma 6. If $c_{r}$ is positive semidefinite and $\alpha<0$, then the Riccati equation in $F$ has a finite solution for any finite $T$.

A closed form solution can easily be obtained in the infinite horizon version of this model $(T=\infty)$, if, for example, $c_{r}, b_{\eta}, \Sigma$, and $\kappa$ are diagonal. A simple solution (with finite $T$ ) is also obtained if $c_{r}=b_{\eta}=0$, implying that $r$ is an Ornstein-Uhlenbeck process and $\eta$ is constant. In this case $Z$ and $\sigma_{t}^{R^{\prime}} \psi$ are deterministic, $F=0$, and

$$
D_{t}=-\left(\int_{t}^{T} e^{-\kappa^{\prime}(s-t)} e^{-\beta(s-t)}\left(k_{s}^{-1}-1\right) d s\right) k_{t} b_{r} .
$$

\subsection{The Additive Case}

In this Section, we assume that $f$ is given by (8) with $\alpha=0$ and Case 3 of Section 5.4 therefore applies. Since the solution method is analogous to the $\gamma=0$ case above, we briefly outline the approach, referring to Schroder and Skiadas [45] for further details.

For Model A, we conjecture a solution to (24) of the form $p(\tau, y)=$ $G_{\tau}+H_{\tau} \cdot y$, where $H$ and $G$ are functions of time valued in $\mathbb{R}^{n}$ and $\mathbb{R}$, respectively. Direct computation shows that $H$ and $G$ satisfy an ODE system analogous to the corresponding system for $\gamma=0$ above. Moreover, Lemma 5 (with analogous proof) remains valid if the assumptions $\gamma=0$ and $\alpha<0$ are replaced with $\gamma<0$ and $\alpha=0$. A closed form solution (given in Schroder and Skiadas [45]) is easily obtained if $\zeta, \kappa, \Sigma$ are diagonal, $b_{i}, \zeta_{i i}, \kappa_{i i} \geqslant 0$, and $\beta>0$.

For Model B, (24) is solved by a function of the form $p(\tau, y)=$ $C_{\tau}+D_{\tau}^{\prime} y+y^{\prime} F_{\tau} y$, with $F$ symmetric, and where $C, D$, and $F$ are deterministic functions of time satisfying an ODE system analogous to the corresponding system for $\gamma=0$ above. In many cases, the Riccati equation in $F$ can be solved analytically using a procedure described by Gelb [27, Sect. 4.6]. Moreover, Lemma 6 (with analogous proof) remains valid if the assumptions $\gamma=0$ and $\alpha<0$ are replaced with $\gamma<0$ and $\alpha=0$. A particularly simple solution is obtained if $c_{r}=b_{\eta}=0$, in which case $F=0$. A closed form solution (given in Schroder and Skiadas [45]) is also easily obtained if $c_{r}, b_{\eta}, \Sigma$, and $\kappa$ are diagonal, and $\kappa, c_{r} \geqslant 0$.

\section{INTRODUCING A BEQUEST FUNCTION}

We conclude the main part of this paper with an outline of how to incorporate a bequest function in our earlier analysis. We illustrate with a parametric example that has the interesting property that the optimal 
portfolio is instantaneously mean-variance efficient under any price dynamics, while the optimal consumption-to-wealth ratio is typically stochastic. This type of solution has been discussed previously in a discretetime setting by Giovannini and Weil [29]. We will keep our discussion short by omitting existence and verification arguments, as well as most technical details.

\subsection{General Formulation}

We modify our earlier setting, by endowing $D$ with the new inner product

$$
\langle x, y\rangle=E\left[\int_{0}^{T} x_{t} y_{t} d t+x_{T} y_{T}\right]
$$

(In particular, $x=y$ in $D$ implies that $x_{T}=y_{T}$ a.s.) Given consumption plan $c \in \mathscr{C} \subseteq D^{+}$, we interpret $c_{T}$ as a lump sum terminal consumption.

The utility process $V(c)$ is defined as a unique solution to the backward SDE

$$
V_{t}(c)=E_{t}\left[\int_{t}^{T} f\left(s, c_{s}, V_{s}(c)\right) d s+v\left(c_{T}\right)\right], \quad t \in[0, T]
$$

for some function $v: \mathbb{R}^{+} \rightarrow \mathbb{R}$. We assume that the utility function $V_{0}: \mathscr{C} \rightarrow \mathbb{R}$ is strictly increasing. The corresponding utility gradient can be written (under technical assumptions) as $\nabla V_{0}(c ; h)=\langle m(c), h\rangle$, where $m_{t}$ is given by (3) for $t<T$, and

$$
m_{T}(c)=\exp \left(\int_{0}^{T} f_{v}\left(s, c_{s}, V_{s}(c)\right) d s\right) \dot{v}\left(c_{T}\right)
$$

where $\dot{v}$ denotes the derivative of $v$.

Taking as given an Arrow-Debreu state price density $\pi \in D^{++}$, the agent's problem is, as before, to maximize $V_{0}(c)$ subject to the budget feasibility constraint $\langle\pi, c\rangle \leqslant w$. Assuming, for simplicity, that $c \in D^{++}$, the first-order condition for optimality of $c$ is $m(c)=\lambda \pi$ for some $\lambda>0$, together with the budget feasibility constraint. The solution method of this system is analogous to our earlier analysis, with the main new element being the boundary condition corresponding to the fact that $V_{T}(c)=v\left(c_{T}\right)$. We leave all details to the interested reader, and we proceed with an outline of an interesting example. 


\subsection{A Homothetic Example}

The parametric SDU form that we analyze in the remainder of this section is

$$
V_{t}=E_{t}\left[\int_{t}^{T}\left(\frac{c_{s}^{\gamma}}{\gamma} \exp \left(-\gamma V_{s}\right)-\frac{\beta}{\gamma}\right) d s+\log \left(c_{T}\right)+\frac{\delta}{\gamma}\right], \quad t \in[0, T],
$$

where $\beta \geqslant 0,0 \neq \gamma<1$, and $\delta \in \mathbb{R}$. The ordinally equivalent utility process $\hat{V}_{t}=\gamma^{-1} \exp \left(\gamma V_{t}\right)$ satisfies the more suggestive recursion

$$
\hat{V}_{t}=E_{t}\left[\int_{t}^{T} e^{-\beta(s-t)}\left(\frac{c_{s}^{\gamma}}{\gamma} d s-\frac{1}{2} \hat{V}_{s}^{-1} d[\hat{V}]_{s}\right)+e^{-\beta(T-t)+\delta}\left(\frac{c_{T}^{\gamma}}{\gamma}\right)\right],
$$

where $[V]$ again represents the quadratic variation process of $V$. Apart from the bequest function (which can be made arbitrarily small by decreasing $\delta$ ), this corresponds to our earlier homothetic expression, (10), with $\gamma \neq 0$ and $\alpha=-1$. (In Theorem 4 we assumed $\alpha>-1$ in order to avoid having $V_{T}=-\infty$, corresponding to $\delta=-\infty$ in the above formulation.)

What is interesting about this case is that a consumption plan satisfying the first-order conditions of optimality can be financed by an instantaneously mean-variance efficient portfolio (no matter what the state price dynamics are). To see that, we note that, since $f_{v}=-c f_{c}$, the first-order conditions can be rearranged to

$$
\begin{aligned}
-\frac{d}{d t} \exp \left(\int_{0}^{t} f_{v}\left(c_{s}, V_{s}\right) d s\right) & =\lambda \pi_{t} c_{t} \quad \text { and } \\
\exp \left(\int_{0}^{T} f_{v}\left(c_{s}, V_{s}\right) d s\right) & =\lambda \pi_{T} c_{T} .
\end{aligned}
$$

It follows that the wealth process, $W$, corresponding to $c$ satisfies

$$
\pi_{t} W_{t}=E_{t}\left[\int_{t}^{T} \pi_{s} c_{s} d s+\pi_{T} c_{T}\right]=\frac{1}{\lambda} \exp \left(\int_{0}^{t} f_{v}\left(c_{s}, V_{s}\right) d s\right) .
$$

Therefore, $\pi_{t} W_{t}$ is absolutely continuous, which is equivalent to the condition $\sigma^{W}=\eta^{\prime}$. Arguing as in Section 2.3, it follows that the optimal portfolio allocation is the same as that of an investor with time-additive logarithmic utility (given in Theorem 2(c) with $k_{t}=1$ ).

The optimal consumption-to-wealth ratio is given by

$$
\frac{c_{t}}{W_{t}}=\exp \left(-\frac{\gamma J_{t}}{1-\gamma}\right), \quad t \in[0, T),
$$


where the process $J$ solves ${ }^{11}$

$$
J_{t}=E_{t}\left[\int_{t}^{T} \frac{1-\gamma}{\gamma} \exp \left(-\frac{\gamma J_{s}}{1-\gamma}\right)-\frac{\beta}{\gamma}+r_{s}+\frac{\eta_{s} \cdot \eta_{s}}{2} d s+\frac{\delta}{\gamma}\right]
$$

The PDE characterization of $J$ in a Markovian setting, and examples analogous to those of Section 6 are left to the reader.

We conclude with an outline of a proof of the above claim, omitting several technical details. Let us first define the process $X$ as the solution to (4a), with

$$
V_{t}=J_{t}-X_{t} \quad \text { and } \quad I(t, x, v)= \begin{cases}\exp ((x+\gamma v) /(\gamma-1)), & \text { if } t<T ; \\ \exp (-x), & \text { if } t=T .\end{cases}
$$

With $(X, V)$ thus defined, we claim that the optimal consumption plan is given by

$$
c_{t}=I\left(t, X_{t}, V_{t}\right)= \begin{cases}\exp \left(-X_{t}-\gamma J_{t} /(1-\gamma)\right), & \text { if } t \in[0, T) \\ \exp \left(-X_{T}\right), & \text { if } t=T,\end{cases}
$$

where $X_{0}=\log (\lambda)$ has been chosen to make the budget constraint tight. (We will see later that $\lambda=-w$.) To show that, suppose for the moment that $V$ is in fact the utility process, $V(c)$, corresponding to $c$. Equation (27) is equivalent to $f_{c}\left(c_{t}, V_{t}\right)=\exp \left(X_{t}\right)$ for $t<T$, and $\dot{v}\left(c_{T}\right)=\exp \left(X_{T}\right)$. On the other hand, Eq. (4a) gives

$$
\exp \left(X_{t}\right)=\lambda \pi_{t} \exp \left(-\int_{0}^{t} f_{v}\left(c_{s}, V_{s}\right) d s\right)
$$

This confirms the first-order condition $m=\lambda \pi$, which (by an omitted verification argument) implies optimality. To confirm that $V=V(c)$, we notice that

$$
-f_{v}\left(c_{t}, V_{t}\right)=\gamma f\left(c_{t}, V_{t}\right)+\beta=\exp \left(-\frac{\gamma J_{t}}{1-\gamma}\right)
$$

The drift term of $J$ is therefore equal to $-\left(f_{v}+r+\eta \cdot \eta / 2\right)-f$, which together with (4a) implies that the drift term of $d V_{t}+f d t$ vanishes. It

${ }^{11}$ For the sake of notational simplicity, our choice of $J$ here is not consistent with that in Section 5.3. It can easily be shown that $\hat{J}_{t}=\exp \left(\gamma J_{t} /(1-\gamma)\right)$ is the first component of the solution to a backward SDE analogous to (18). 
follows that $d V_{t}=-f\left(c_{t}, V_{t}\right) d t+d M_{t}$, for some martingale $M$, and since $V_{T}=(\delta / \gamma)-X_{T}=V_{T}(c), V=V(c)$.

Finally, Eqs. (26) and (28) give $W_{t}=\exp \left(-X_{t}\right.$ ) (and therefore $\lambda=-w$ ). Combining this with (27) results in the claimed consumption-to-wealth ratio formula. The optimal consumption dynamics can also be easily obtained by applying Ito's lemma to (27).

\section{APPENDIX A}

\section{A Class of Backward SDE}

In this appendix we prove existence, uniqueness, monotonicity, and convexity properties for a class of backward SDE that arise in connection with the homothetic specification of SDU discussed in Section 3.

The backward SDEs we will consider are of the form

$$
d V_{t}=-\left(U_{t}-\beta_{t} V_{t}+\frac{1}{2} A\left(V_{t}\right)\left\|Z_{t}\right\|^{2}\right) d t+Z_{t} d B_{t}, \quad V_{T}=0,
$$

to be solved for an adapted pair $(V, Z)$. Theorem 1 and the analysis of Section 5 require the solution of the above backward SDE with either $A\left(V_{t}\right)$ or $A\left(V_{t}\right) V_{t}$ being a deterministic constant (and proper restrictions on $U$ and $\beta$ ). We now consider these two cases in turn. The terminal value $V_{T}$ has been set to zero for brevity of exposition and since this is the case we focus on in the main text. The mathematical arguments, however, extend readily to incorporate a more general terminal value. In fact, in some cases it will become apparent that the zero-terminal-value case is mathematically the most difficult one, due to integrability concerns as time approaches $T$.

Throughout this appendix, the underlying probability space, the $n$-dimensional Brownian motion, $B$, and associated filtration, and the space $D$ (with its positive cone $D^{+}$and strictly positive cone $D^{++}$) are as in Section 2. $D^{n}$ denotes the Cartesian product of $n$ copies of $D$. For any $Z \in D^{n}$, each value, $Z(\omega, t)$, should be thought of as a $n$-dimensional row vector with Euclidean norm $\|Z(\omega, t)\|$. (In particular, $\|\cdot\|$ does not denote the norm induced by the inner product of $D$.) In addition to the set $D_{0}$ of Section 4.2 , the following subsets of $D$ will be used:

$$
\begin{aligned}
D_{1} & =\left\{X \in D: E\left[\int_{0}^{T}\left|X_{t}\right|^{l} d t\right]<\infty, \text { for every } l \in(0, \infty)\right\}, \\
D_{0}^{\exp } & =\left\{X \in D: E\left[\exp \left(\operatorname{ess} \sup _{t} l\left|X_{t}\right|\right)\right]<\infty, \text { for every } l \in(-\infty, \infty)\right\} . \\
D_{1}^{\exp } & =\left\{X \in D: E\left[\exp \left(l \int_{0}^{T}\left|X_{t}\right| d t\right)\right]<\infty, \text { for every } l \in(-\infty, \infty)\right\} .
\end{aligned}
$$


For any $S \subset D$, we define $S^{+}=S \cap D^{+}$and $S^{++}=S \cap D^{++}$. As always, we identify processes that are modifications of each other.

\section{The Case of Constant $A(V)$}

This section is on the backward SDE (A1) under the assumption that $A$ is identically equal to some constant $\alpha, U \in D_{1}^{\exp }$, and $\beta \in D$ is non-negative and bounded. For $\alpha=0$, one obtains easily the solution: $V_{t}=E_{t}\left[\int_{t}^{T} U_{s} \exp \right.$ $\left.\left(-\int_{t}^{s} \beta_{u} d u\right) d s\right]$. Assuming $\alpha \neq 0$, a simple rescaling shows that we can assume without loss of generality that $\alpha=1$. Our objective in this section is therefore to analyze the backward SDE

$$
d V_{t}=-\left(U_{t}-\beta_{t} V_{t}+\frac{1}{2}\left\|Z_{t}\right\|^{2}\right) d t+Z_{t} d B_{t}, \quad V_{T}=0 .
$$

The following is the central result of this section.

THeorem A1. Suppose that $U \in D_{1}^{\exp }$, and $\beta \in D^{+}$is non-negative and bounded. Then there exists a unique pair $(V, Z) \in D_{0}^{\exp } \times D^{n}$ satisfying (A2). Moreover, the solution $V$ as a function of the parameter $U$ is monotonically increasing and convex.

The proof of Theorem A1 will proceed in a sequence of lemmas. We assume throughout that $U \in D_{1}^{\exp }$ and that, for some $\bar{\beta} \in \mathbb{R}, 0 \leqslant \beta_{t} \leqslant \bar{\beta}$ for all $t$.

We begin with a convenient reformulation of the problem. For any $(V, Z) \in D_{0}^{\exp } \times D^{n}$ and stopping time $\tau$, we consider the recursion

$$
V_{t}=\log \left(E_{t}\left[\exp \left(\int_{t}^{\tau} U_{s}-\beta_{s} V_{s} d s+V_{\tau}\right)\right]\right) \quad \text { on }\{\tau \geqslant t\} .
$$

Lemma A1. The following statements are equivalent, for any $V \in D_{0}^{\exp }$ :

(a) There exists a unique $Z \in D^{n}$ such that $(V, Z)$ satisfies (A2).

(b) There exists some $Z \in D^{n}$ such that $(V, Z)$ satisfies (A2).

(c) $V$ satisfies (A3) with $\tau=T$, and $V_{T}=0$.

(d) $V$ satisfies (A3) for any stopping time $\tau$, and $V_{T}=0$.

Proof. (d) $\Leftrightarrow(\mathrm{c})$. Clearly, (c) is implied by (d). Conversely, suppose that (c) holds. We then have

$$
\exp \left(V_{t}\right)=\exp \left(-\int_{0}^{t} U_{s}-\beta_{s} V_{s} d s\right) M_{t}
$$

where $M$ is the martingale defined by

$$
M_{t}=E_{t}\left[\exp \left(\int_{0}^{T} U_{s}-\beta_{s} V_{s} d s\right)\right]
$$


Suppose now that $\tau$ is any stopping time. By the optional sampling theorem, $M_{t}=E_{t}\left[M_{\tau}\right]$ on $\{\tau \geqslant t\}$. From (A4), we have

$$
\exp \left(\int_{t}^{\tau} U_{s}-\beta_{s} V_{s} d s+V_{\tau}\right)=\exp \left(-\int_{0}^{t} U_{s}-\beta_{s} V_{s} d s\right) M_{\tau} \quad \text { on }\{\tau \geqslant t\} .
$$

Applying the operator $E_{t}$ on both sides, simplifying the right-hand side, and using (A4) again, (d) follows.

(c) $\Rightarrow$ (b). Suppose that (c) holds, and let $M$ be the martingale defined by (A5), so that (A4) is also valid. By the martingale representation theorem, there exists a unique predictable $\hat{Z} \in D^{n}$ such that $d M_{t}=\hat{Z}_{t} d B_{t}$. Let the process $Z$ be defined by $Z_{t}=\hat{Z}_{t} M_{t}^{-1}$. Suppose, for now, that $Z \in D^{n}$. Letting $W_{t}=\exp \left(V_{t}\right)$, and applying integration by parts to (A4), we obtain

$$
\frac{d W_{t}}{W_{t}}=-\left(U_{t}-\beta_{t} V_{t}\right) d t+Z_{t} d B_{t}
$$

Finally, (A2) follows from an application of Ito's lemma to $V_{t}=\log \left(W_{t}\right)$.

To conclude that (b) holds, however, we still need to show that $Z \in D^{n}$, which we do next. Let $N_{t}=M_{t}^{-1}$ and $N^{*}=\max _{t} N_{t}$. From the definition of $Z$ and the Cauchy-Schwarz inequality, we have

$$
\begin{aligned}
\left(E\left[\int_{0}^{T}\left\|Z_{t}\right\|^{2} d t\right]\right)^{2} & \leqslant\left(E\left[\left(N^{*}\right)^{2} \int_{0}^{T}\left\|\hat{Z}_{t}\right\|^{2} d t\right]\right)^{2} \\
& \leqslant E\left[\left(N^{*}\right)^{4}\right] E\left[\left(\int_{0}^{T}\left\|\hat{Z}_{t}\right\|^{2} d t\right)^{2}\right] .
\end{aligned}
$$

To show that $Z \in D^{n}$, it suffices to show that the above expression is finite. By the Burkholder-Davis-Gundy inequalities, $\int_{0}^{T}\left\|\hat{Z}_{t}\right\|^{2} d t$ has a finite second moment if $M_{T}$ has a finite fourth moment, which it does since $V \in D_{0}^{\exp }$ and $U \in D_{1}^{\exp }$. To show that $N^{*}$ has finite fourth moment, we first notice that, by Jensen's inequality, $N$ is a submartingale. By Doob's maximal inequality and (A5), there exists some constant $C$ such that

$$
E\left[\left(N^{*}\right)^{4}\right] \leqslant C E\left[N_{T}^{4}\right]=C E\left[\exp \left(-4 \int_{0}^{T} U_{s}-\beta_{s} V_{s} d s\right)\right] .
$$

The latter is finite since $V \in D_{0}^{\exp }$ and $U \in D_{1}^{\exp }$, and the proof that $Z \in D^{n}$ is complete.

(b) $\Rightarrow$ (c). Suppose that $(V, Z) \in D_{0}^{\exp } \times D^{n}$ satisfies (A2), and define $W_{t}=\exp \left(V_{t}\right)$. By Ito's lemma, $W$ satisfies (A6). Let $M$ be the stochastic 
exponential of $Z$, that is, the unique local martingale that satisfies $d M_{t}=M_{t} Z_{t} d B_{t}, M_{0}=1$. (As is well known, stronger restrictions on $Z$ than mere membership to $D^{n}$ are required to make $M$ a martingale.) Integration by parts then gives (A4). Given any stopping time $\tau$, we let $M_{t}^{\tau}=M_{t} 1_{\{t \leqslant \tau\}}+M_{\tau} 1_{\{t>\tau\}}$. Let $\{\tau(n)\}$ be an increasing sequence of stopping times that converges to $T$, such that the stopped process $M^{\tau(n)}$ is a martingale, for every $n$. Arguing as in the first part of the proof (where (A3) was derived from (A4) and the martingale property of $M$ ), we have

$$
\exp \left(V_{t}\right)=E_{t}\left[\exp \left(\int_{t}^{\tau(n)} U_{s}-\beta_{s} V_{s} d s+V_{\tau(n)}\right)\right] \quad \text { on }\{\tau(n) \geqslant t\} .
$$

Letting $n \rightarrow \infty$ and using the dominated convergence theorem (made possible by the assumption $V \in D_{0}^{\exp }$ and $U \in D_{1}^{\exp }$ ), (c) follows. Having shown (c), it follows that $M$ is given by (A5), and it is after all a true martingale.

(b) $\Leftrightarrow$ (a). Clearly, (a) implies (b). Suppose that (b) holds, and let $W_{t}=\exp \left(V_{t}\right)$. Then $Z$ represents the diffusion term of the Ito decomposition of $d W_{t} / W_{t}$ and is therefore uniquely determined in $D$.

An interesting corollary of Lemma A1 is that for $\beta=0$, (A3) (with $\tau=T$ ) gives a closed-form expression for the $V \in D_{0}^{\exp }$ that is part of the unique solution to (A2). For the case of nonzero $\beta$, however, a closed-form expression is not apparent to us, and we will resort to a fixed-point argument. For this purpose, we define a function $F_{U}: D_{0}^{\exp } \rightarrow D_{0}^{\exp }$, corresponding to the parameter process $U$, as follows:

$$
F_{U}(V)_{t}=\log \left(E_{t}\left[\exp \left(\int_{t}^{T} U_{s}-\beta_{s} V_{s} d s\right)\right]\right), \quad t \in[0, T]
$$

(That $F_{U}$ is indeed valued in $D_{0}^{\exp }$ can easily be confirmed using Doob's maximal inequality.) By Lemma A1, we are interested in finding a unique fixed point of the function $F_{U}$ and in showing the monotonicity of the fixed point as a function of the parameter $U$. Assuming existence for now, uniqueness and monotonicity will be consequences of the following result:

Lemma A2. Suppose that, for some $U \in D_{1}^{\exp }$, the following conditions hold:

(a) $V \in D_{0}^{\exp }$ satisfies $V=F_{U}(V)$.

(b) $\widetilde{V} \in D_{0}^{\exp }$ is continuous, $\widetilde{V}_{T} \geqslant 0$, and, for any stopping time $\tau$,

$$
\tilde{V}_{t} \geqslant \log \left(E_{t}\left[\exp \left(\int_{t}^{\tau} U_{s}-\beta_{s} \tilde{V}_{s} d s+\tilde{V}_{\tau}\right)\right]\right) \quad \text { on }\{\tau>t\}, \quad t \in[0, T] \text {. }
$$


Then $\tilde{V}_{t} \geqslant V_{t}$, for all $t \in[0, T]$. The result also holds with all inequalities reversed.

Proof. Suppose that, for some $t$, the event $A=\left\{\widetilde{V}_{t}<V_{t}\right\}$ is non-null. Consider the stopping time

$$
\tau=\inf \left\{s \geqslant t: \widetilde{V}_{s} \geqslant V_{s}\right\} .
$$

Since almost all paths of $\widetilde{V}$ and $V$ are continuous and $\widetilde{V}_{T} \geqslant 0=V_{T}$, we have $\widetilde{V}_{\tau}=V_{\tau}$ on $A$, while $\widetilde{V}_{s}<V_{s}$ on $A \cap\{t \leqslant s<\tau\}$. By our hypotheses and Lemma A1, we have, on event $A$,

$$
\begin{aligned}
0> & \exp \left(\tilde{V}_{t}\right)-\exp \left(V_{t}\right) \\
\geqslant & E_{t}\left[\exp \left(\int_{t}^{\tau} U_{s}-\beta_{s} \tilde{V}_{s} d s+\tilde{V}_{\tau}\right)\right. \\
& \left.\quad-\exp \left(\int_{t}^{\tau} U_{s}-\beta_{s} V_{s} d s+V_{\tau}\right)\right] \geqslant 0,
\end{aligned}
$$

a contradiction. The same argument applies with all inequalities reversed.

Suppose now that, for each $i \in\{1,2\}, U^{i} \in D_{1}^{\exp }$, and $V^{i} \in D_{0}^{\exp }$ satisfies $F_{U^{i}}\left(V^{i}\right)=V^{i}$. An immediate consequence of the last two lemmas is that if $U^{1} \geqslant U^{2}$, then $V^{1} \geqslant V^{2}$, proving the monotonicity claim of Theorem A1. Moreover, taking $U^{1}=U^{2}=U$, it follows that any two fixed points, $V^{1}$ and $V^{2}$, of $F_{U}$ must satisfy both $V^{1} \geqslant V^{2}$ (since $U^{1} \geqslant U^{2}$ ) and $V^{2} \geqslant V^{1}$ (since $U^{2} \geqslant U^{1}$ ). Therefore $V^{1}=V^{2}$, proving that $F_{U}$ has at most one fixed point.

Next, we turn to the question of existence of a fixed point of $F_{U}$. We begin with the special case of a bounded $U$. Let $\mathscr{B}$ be the space of progressively measurable bounded processes, metrized by the (pseudo)metric

$$
d(x, y)=\text { ess } \sup _{(\omega, t)}|x(\omega, t)-y(\omega, t)|, \quad x, y \in \mathscr{B} .
$$

As usual, we identify any two processes $x, y \in \mathscr{B}$ such that $d(x, y)=0$, which makes $(\mathscr{B}, d)$ a complete metric space. $\mathscr{B}$ is ordered in the usual sense: $x \geqslant y$ means $P\left[x_{t} \geqslant y_{t}\right]=1$ for all $t$.

\section{Lemma A3. If $U \in \mathscr{B}$, then $F_{U}(V)=V$ for some $V \in \mathscr{B}$.}

Proof. Since $U$ is fixed throughout, we simplify notation by letting $F=F_{U}$. We use Blackwell's version of the contraction fixed-point theorem (see, for example, Theorem 3.3 of Stokey and Lucas [48]) to show that $F$ is a contraction if $\bar{\beta} T<1$ (recall that $\bar{\beta}$ is an upper bound of $\beta$ ). This partial 
result will then be generalized by partitioning the time horizon and by piecing together a solution backward in time. Alternatively, for any $T$, we can show that $F$ composed with itself $k$ times is a contraction for sufficiently large $k$.

Given any real number $x$, we denote also by $x$ the function in $\mathscr{B}$ identically equal to $x$. From the functional form of $F$ it follows easily that, for every $V \in \mathscr{B}$ and positive real $x, F(V+x) \geqslant F(V)-\bar{\beta} T x$. Consider now any $V, W \in \mathscr{B}$. Since $V \leqslant W+d(V, W)$ and $F$ is decreasing, we have $F(V) \geqslant$ $F(W+d(V, W)) \geqslant F(W)-\bar{\beta} T d(V, W)$. Interchanging the roles of $V$ and $W$, we also have $F(W) \geqslant F(V)-\bar{\beta} T d(W, V)$. Therefore, $d(F(V), F(W)) \leqslant$ $\bar{\beta} T d(V, W)$, proving that $F$ is a contraction if $\bar{\beta} T<1$.

If $\bar{\beta} T \geqslant 1$, choose any integer $N>\bar{\beta} T$. Initially, set $k=N-1$. The above argument shows that there exists a $V \in \mathscr{B}$ satisfying (A2) for all $t \geqslant(k / N) T$. We proceed inductively. Suppose that the last statement is true for arbitrary $k \in\{1, \ldots, N-1\}$. Let $V \in \mathscr{B}$ be a solution of (A2) over the time horizon $[T k / N, T]$. Applying the same contraction argument over the time interval $[T(k-1) / N, T k / N]$ with terminal value $V_{k T / N}$, the proof of the lemma is easily completed.

Alternatively, we can define $d_{t}(x, y)=\operatorname{ess} \sup _{(\omega, s \geqslant t)}|x(\omega, s)-y(\omega, s)|$. Then, by the argument used above, $d_{t}(F(V), F(W)) \leqslant \bar{\beta}(T-t) d_{0}(V, W)$. A similar argument repeated $k$ times gives

$$
d_{t}\left(F^{(k)}(V), F^{(k)}(W)\right) \leqslant \frac{(\bar{\beta}(T-t))^{k}}{k !} d_{0}(V, W) .
$$

For large enough $k, F^{(k)}$ is therefore a contraction and, for a unique $V \in \mathscr{B}$, $F^{(k)}(V)=V$. Applying $F$ on both sides of this equation, it follows that $F(V)$ is also a fixed point of $F^{(k)}$ and is therefore equal to $V$. This shows that $V$ is a fixed point of $F$.

Using the last two lemmas, we now show that, for any $U \in D_{1}^{\exp }, F_{U}$ has a fixed point in $D_{0}^{\exp }$. Suppose first that $U \in D_{1}^{\exp }$ is bounded below. For every integer $n$, let $U_{t}^{n}=\min \left\{U_{t}, n\right\}$, and let $V^{n} \in \mathscr{B}$ solve $F_{U^{n}}\left(V^{n}\right)=V^{n}$. By our earlier results, $V^{n}$ exists and is monotonically increasing in $n$. Using these facts, we have

$$
V_{t}^{n} \leqslant \log E_{t}\left[\exp \left(\int_{t}^{T} U_{s}-\beta_{s} V_{s}^{1} d s\right)\right]
$$

Since $V^{1} \in D_{0}^{\exp }$, it follows that the sequence $\left\{V^{n}\right\}$ is bounded above almost surely, and therefore there exists some $V$ such that $V_{n} \uparrow V$ a.s. as $n \rightarrow \infty$. Using Doob's maximal inequality, it is also easy to conclude from the 
above bound on $V^{n}$ that $V \in D_{0}^{\exp }$. Letting $n$ go to infinity in $F_{U^{n}}\left(V^{n}\right)=V^{n}$ and using the dominated convergence theorem, we have $F_{U}(V)=V$. This proves the lemma assuming that $U$ is bounded below. To extend the existence proof for $U$ unbounded below, we use an analogous argument with $U^{n}=\max \{U,-n\}$, showing that the corresponding solutions $V^{n}$ converge monotonically from above to a fixed point of $F_{U}$. This completes the proof of existence and monotonicity.

Finally, we prove the convexity of the solution $V$ as a function of the parameter $U$. For $i \in\{a, b\}$, let $U^{i} \in D_{1}^{\exp }$, and let $\left(V^{i}, Z^{i}\right) \in D_{0}^{\exp } \times D^{n}$ satisfy

$$
d V_{t}^{i}=-\left(U_{t}^{i}-\beta_{t} V_{t}^{i}+\frac{1}{2}\left\|Z_{t}^{i}\right\|^{2}\right) d t+Z_{t}^{i} d B_{t}, \quad V_{T}^{i}=0 .
$$

Fixing an arbitrary $v \in(0,1)$, we define $\left(V^{v}, Z^{v}\right)=v\left(V^{a}, Z^{a}\right)+(1-v)$ $\left(V^{b}, Z^{b}\right)$, and we assume that $(V, Z) \in D_{0}^{\exp } \times D^{n}$ is the solution to (A2) corresponding to $U \equiv v U^{a}+(1-v) U^{b}$. Convexity follows, if we can prove that $V_{0}^{v} \geqslant V_{0}$. To this end, we define $2 \Delta_{t}=\left(v\left\|Z_{t}^{a}\right\|^{2}+(1-v)\left\|Z_{t}^{b}\right\|^{2}\right)-$ $\left\|Z_{t}^{v}\right\|^{2}>0$ (positivity follows from Jensen's inequality). It follows that

$$
d V_{t}^{v}=-\left(U_{t}+\Delta_{t}-\beta_{t} V_{t}^{v}+\frac{1}{2}\left\|Z_{t}^{v}\right\|^{2}\right) d t+Z_{t}^{v} d B_{t}, \quad V_{T}^{v}=0 .
$$

Ignoring for now the need for an integrability restriction on $\Delta$, Lemma A1 implies that, for any stopping time $\tau$,

$$
\begin{aligned}
\exp \left(V_{t}^{v}\right) & =E_{t}\left[\exp \left(\int_{t}^{\tau} U_{s}+\Delta_{s}-\beta_{s} V_{s}^{v} d s+V_{\tau}^{v}\right)\right] \\
& >E_{t}\left[\exp \left(\int_{t}^{\tau} U_{s}-\beta_{s} V_{s}^{v} d s+V_{\tau}^{v}\right)\right] .
\end{aligned}
$$

By Lemma A2, we obtain $V_{0}^{v} \geqslant V_{0}$ (and in fact, it is not hard to show that the inequality is strict).

As noted above, the application of Lemma A1 is not justified without appropriate integrability restrictions on $\Delta$, a difficulty that is easily overcome by slightly modifying Lemma A1. Returning to the last part of the proof of Lemma A1 ( (b) $\Rightarrow(\mathrm{c}))$, we make the following two changes: (i) We assume the localizing sequence $\{\tau(n)\}$ converges to a stopping time $\tau$, instead of $T$ (by just taking the minimum of the original sequence elements and $\tau$ ). (ii) By replacing $\Delta$ with $\min \{\Delta, 1\}$ before taking expectations, we conclude that

$$
\begin{gathered}
\exp \left(V_{t}^{v}\right) \geqslant E_{t}\left[\exp \left(\int_{t}^{\tau(n)} U_{s}+\min \left\{\Delta_{s}, 1\right\}-\beta_{s} V_{s}^{v} d s+V_{\tau(n)}^{v}\right)\right], \\
n=1,2, \ldots
\end{gathered}
$$


Taking the limit as $n \rightarrow \infty$, we can now complete the proof as before without the integrability concern. This also completes the proof of Theorem A1.

\section{The Case of Constant $A(V) V$}

In this section we analyze the backward SDE (A1) with the specification $A(v) v$ equal to some constant greater than or equal to minus one, $U \in D_{1}^{++}$, and $\beta \in D_{1}^{\exp }$. Making the change of variable $\hat{V}_{t}=V_{t} \exp$ $\left(-\int_{0}^{t} \beta_{s} d s\right)$, it becomes clear that we can assume without loss in generality that $\beta=0$, which we do. More precisely, we consider the backward SDE

$$
d V_{t}=-\left(U_{t}+\frac{p-1}{2} \frac{\left\|Z_{t}\right\|^{2}}{V_{t}}\right) d t+Z_{t} d B_{t}, \quad t<T, \quad V_{T}=V_{T-}=\varepsilon
$$

where $\varepsilon \geqslant 0, p>0$, and $U \in D_{1}^{++}$. Although for the purposes of the main text we are only interested in the case $\varepsilon=0$, we will attack this case by first solving with a positive $\varepsilon$, and then letting $\varepsilon$ approach zero.

Let $\mathscr{L}^{2}$ be the space of all progressively measurable processes, $Z$, valued in $\mathbb{R}^{n}$, satisfying $\int_{0}^{T}\left\|Z_{t}\right\|^{2} d t<\infty$ a.s. As usual, we identify any two elements $Z, \tilde{Z} \in \mathscr{L}^{2}$ such that $\int_{0}^{T}\left\|Z_{t}-\tilde{Z}_{t}\right\|^{2} d t=0$ a.s. The following is the section's main conclusion

Theorem A2. Suppose that $\varepsilon \in[0, \infty), p \in(0, \infty)$, and $U \in D_{1}^{++}$. Then there exists a unique pair $(V, Z) \in D_{0}^{++} \times \mathscr{L}^{2}$ satisfying (A7). Moreover, the solution $V$ as a function of the parameter $U$ is monotonically increasing, and it is convex if $p \in[1, \infty)$ and concave if $p \in(0,1]$.

In the remainder of this section we prove Theorem A2. The structure of the proof parallels that of Theorem A1, but the details are considerably more delicate. We assume throughout that $\varepsilon \geqslant 0$ and $p>0$.

We begin with the closely related recursion

$$
V_{t}^{p}=E_{t}\left[\int_{t}^{\tau} p U_{s} V_{s}^{p-1} d s+V_{\tau}^{p}\right] \quad \text { on }\{\tau>t\}
$$

where $\tau$ is any stopping time.

Lemma A4. Suppose that $U \in D_{1}^{++}$and $V \in D_{0}^{++}$. Then the following conditions are equivalent:

(a) There exists a unique $Z \in \mathscr{L}^{2}$ such that ( $\left.V, Z\right)$ satisfies (A7).

(b) There exists some $Z \in \mathscr{L}^{2}$ such that $(V, Z)$ satisfies (A7).

(c) $V$ satisfies (A8) with $\tau=T$ and $V_{T}=\varepsilon$.

(d) $V$ satisfies (A8) for any stopping time $\tau$, and $V_{T}=\varepsilon$. 
Proof. The key to the proof lies in the fact that (A7) together with Ito's lemma implies

$$
d V_{t}^{p}=-V_{t}^{p-1} p U_{t} d t+p V_{t}^{p-1} Z_{t} d B_{t}
$$

The details are analogous to Lemma A1. The weaker integrability restriction on $Z$ simplifies part of the proof. On the other hand, in deriving the integral representation from the differential representation, it is necessary to use a localizing stopping time sequence and take a limit (using monotone convergence for the first term, and dominated convergence for the second). The details are left to the reader.

For every $\varepsilon \geqslant 0$ and $U \in D_{1}^{++}$, we define the operator $F_{U}^{\varepsilon}: D_{0}^{++} \rightarrow D_{0}^{++}$by

$$
F_{U}^{\varepsilon}(V)_{t}=\left(E_{t}\left[\int_{t}^{T} p U_{s} V_{s}^{p-1} d s+\varepsilon^{p}\right]\right)^{1 / p} .
$$

That $F_{U}^{\varepsilon}$ is indeed valued in $D_{0}^{++}$is a consequence of Doob's maximal inequality. Because of Lemma A4, we are interested in proving that $F_{U}^{\varepsilon}$ has a unique fixed point. We will prove this, as well as the claimed monotonicity and convexity properties of the fixed point, for $\varepsilon>0$ first, and we will then let $\varepsilon$ approach zero.

We start with two lemmas that are used below, as well as in the proof of Theorem 4.

Lemma A5. Suppose that $V=F_{U}^{\varepsilon}(V)$ for some $\varepsilon \in[0,1]$ and $U \in D_{1}^{++}$. Then, there exists some positive constant $K$, such that

$$
E_{t}\left[\int_{t}^{T} U_{s} d s\right] \leqslant V_{t} \leqslant K\left(E_{t}\left[\int_{t}^{T} U_{s}^{p} d s+1\right]\right)^{1 / p} \quad \text { if } \quad p \in(1, \infty),
$$

and

$$
K\left(E_{t}\left[\int_{t}^{T} U_{s}^{p} d s\right]\right)^{1 / p} \leqslant V_{t} \leqslant E_{t}\left[\int_{t}^{T} U_{s} d s+1\right] \quad \text { if } \quad p \in(0,1) .
$$

For $p=1$, we have $V_{t}=E_{t}\left[\int_{t}^{T} U_{s} d s\right]$.

Proof. The case $p=1$ is immediate from Lemma A4. Suppose now that $p>1$. By Lemma A4,

$$
V_{t}=E_{t}\left[\int_{t}^{T}\left(U_{s} d s+\frac{p-1}{2} \frac{\left(d V_{t}\right)^{2}}{V_{t}}\right)+\varepsilon\right] \geqslant E_{t}\left[\int_{t}^{T} U_{s} d s+\varepsilon\right] .
$$


To derive the right-hand inequality, we let $\rho=(p-1) / p \in(0,1)$ and use the gradient inequality $x^{\rho} \leqslant 1+\rho(x-1), \quad x \in \mathbb{R}$. In particular, letting $x=\left(V_{t} / U_{t}\right)^{p}$ and rearranging, we obtain $p U_{t} V_{t}^{p-1} \leqslant U_{t}^{p}+(p-1) V_{t}^{p}$. Therefore,

$$
V_{t}^{p}=E_{t}\left[\int_{t}^{T} p U_{s} V_{s}^{p-1} d s+\varepsilon^{p}\right] \leqslant E_{t}\left[\int_{t}^{T} U_{s}^{p}+(p-1) V_{s}^{p} d s+\varepsilon^{p}\right] .
$$

Lemma C1 implies

$$
V_{t}^{p} \leqslant E_{t}\left[\int_{t}^{T} e^{(p-1)(s-t)} U_{s}^{p} d s+\varepsilon^{p} e^{(p-1)(T-t)}\right] .
$$

Finally, for $p<1$, all the above inequalities are reversed.

Lemma A6. Suppose that $U \in D_{1}^{++}, V, \tilde{V} \in D_{0}^{++}, \tilde{V}$ is continuous, $\varepsilon, \tilde{\varepsilon} \in \mathbb{R}$, and if $p \in(1, \infty)$, then $\tilde{\varepsilon}>0$. If $V=F_{U}^{\varepsilon}(V), \widetilde{V}_{T}=\tilde{\varepsilon} \geqslant \varepsilon \geqslant 0$, and, for any stopping time $\tau$,

$$
\tilde{V}_{t} \geqslant\left(E_{t}\left[\int_{t}^{\tau} p U_{s} \tilde{V}_{s}^{p-1} d s+\tilde{V}_{\tau}^{p}\right]\right)^{1 / p} \quad \text { on }\{\tau>t\}, \quad t \in[0, T],
$$

then $\tilde{V}_{t} \geqslant V_{t}$ for all $t \in[0, T]$.

Proof. Suppose first that $p \in(0,1)$. Utilizing the gradient inequality applied to the convex function $x \mapsto x^{(p-1) / p}$, we obtain, for any stopping time $\tau$,

$$
\begin{aligned}
\tilde{V}_{t}^{p}-V_{t}^{p} & \geqslant E_{t}\left[\int_{t}^{\tau} p U_{s}\left(\tilde{V}_{s}^{p-1}-V_{s}^{p-1}\right) d s+\tilde{V}_{\tau}^{p}-V_{\tau}^{p}\right] \\
& \geqslant E_{t}\left[\int_{t}^{\tau}(p-1) \frac{U_{s}}{V_{s}}\left(\tilde{V}_{s}^{p}-V_{s}^{p}\right) d s+\tilde{V}_{\tau}^{p}-V_{\tau}^{p}\right] .
\end{aligned}
$$

The result now follows from Lemma C2. The proof is immediate for $p=1$. Finally, we consider the case of $p>1$. Again using the gradient inequality applied to the now concave function $x \mapsto x^{(p-1) / p}$, we obtain, for any stopping time $\tau$,

$$
V_{t}^{p}-\tilde{V}_{t}^{p} \leqslant E_{t}\left[\int_{t}^{\tau}(p-1) \frac{U_{s}}{\tilde{V}_{s}}\left(V_{s}^{p}-\tilde{V}_{s}^{p}\right) d s+V_{\tau}^{p}-\tilde{V}_{\tau}^{p}\right],
$$

and the result follows from Lemma $\mathrm{C} 3$. The required integrability restriction in applying Lemma C3 is satisfied, because $U \in D_{1}$ and $\widetilde{V} \geqslant \tilde{\varepsilon}>0$. 
The above lemma immediately implies that if $U^{1}, U^{2} \in D_{1}^{++}, F_{U^{i}}^{\varepsilon^{i}}\left(V^{i}\right)=$ $V^{i}$ for $i \in\{1,2\}$, and $\left(U^{1}, \varepsilon^{1}\right) \geqslant\left(U^{2}, \varepsilon^{2}\right)>(0,0)$, then $V^{1} \geqslant V^{2}$. It also implies that $F_{U}^{\varepsilon}$ has at most one fixed point in $D_{0}^{++}$if $U \in D_{1}^{++}$and $\varepsilon>0$.

Next we argue that to prove Theorem A2 it suffices to show the case of positive terminal value. To see that, suppose that $V[\varepsilon] \in D_{0}^{++}$is the unique fixed point of $F_{U}^{\varepsilon}$ for $\varepsilon>0$, and the dependence of $V[\varepsilon]$ on $U$ is monotone and convex (monotonicity and uniqueness were proved in the last paragraph). Recalling that $V[\varepsilon]$ is decreasing in $\varepsilon$, we define $V_{t}=$ $\lim _{\varepsilon \downarrow 0} V[\varepsilon]_{t}$. By Lemma A5 and dominated convergence, $V \in D_{0}^{++}$is a fixed point of $F_{U}^{0}$. Moreover, $V$ inherits the monotone and convex dependence on $U$. It remains to show that $V$ is the unique fixed point of $F_{U}^{0}$. For $p \in(0,1]$ this is immediate from Lemma A6, but the argument does not apply with $p>1$ (because the integrability restrictions required by Lemma C3 are violated). Instead, the following indirect argument applies. Suppose that $\hat{V}$ is another fixed point of $F_{U}^{0}$. For any $\varepsilon>0$, Lemma A6 shows that $V[\varepsilon] \geqslant \hat{V}$. Letting $\varepsilon$ approach zero proves that $V \geqslant \hat{V}$. To show the reverse inequality, we confirm that $V \leqslant \hat{V}+\varepsilon$ for any $\varepsilon>0$. Fixing $\varepsilon>0$, let $\widetilde{V}=\hat{V}+\varepsilon$. Applying Ito's lemma to $\widetilde{V}^{p}$ (as in Lemma A4), it follows that the assumption of Lemma A6 is satisfied and $\widetilde{V} \geqslant V$. This completes the proof of uniqueness.

Given the above arguments, we assume that $\varepsilon>0$ throughout the remainder of this proof. We first show that $F_{U}^{\varepsilon}$ has a fixed point. Because of homogeneity, it is sufficient to prove the $F_{U}^{1}$ has a fixed point. We consider the bounded case first

Lemma A7. Suppose that there exist constants $k, K$ such that $0<k<U_{t}<K, t \in[0, T]$. Then $F_{U}^{1}$ has a fixed point in $D_{0}^{++}$, which is also bounded from above and away from zero.

Proof. We let the metric space of progressively measurable bounded processes $(\mathscr{B}, d)$ be defined as in the last section, and we introduce the following change of variables:

$$
X_{t}=\log \left(p U_{t}\right), \quad Y_{t}=p \log \left(V_{t}\right), \quad \rho=\frac{p-1}{p}<1 .
$$

The lemma's hypothesis implies that $X \in \mathscr{B}$. The fixed-point condition $V=F_{U}^{1}(V)$ can be written as

$$
Y_{t}=\log \left(E_{t}\left[\int_{t}^{T} \exp \left(X_{s}+\rho Y_{s}\right) d s+1\right]\right), \quad t \in[0, T] .
$$

The lemma will be proved if we can construct a $Y \in \mathscr{B}$ satisfying (A9). For $\rho \in(-1,1)$, Blackwell's theorem (see, for example, Theorem 3.3 of Stokey 
and Lucas [48]) applies to the operator of Eq. (A9) directly, proving the lemma for this case.

Next we prove by induction the following statement, for any integer $k$ : If $1>\rho>-k$, then there is a unique solution $Y \in \mathscr{B}$ to (A9), for any $X \in \mathscr{B}$. For $k=1$, we have already proved the result. Suppose now we have shown the result for some $k$, and we wish to prove it under the assumption $-k \geqslant \rho>-k-1$. We break the fixed-point problem into two parts

$$
Y_{t}=Z_{t}=\log \left(E_{t}\left[\int_{t}^{T} \exp \left(X_{s}-(1-\varepsilon) Y_{s}+(\rho+1-\varepsilon) Z_{s}\right) d s\right]+1\right),
$$

where $\varepsilon \in(0,1)$ is chosen small enough so that $\rho+1-\varepsilon>-k$. By the induction hypothesis, for any choice of $Y \in \mathscr{B}$, there exists a corresponding $Z \in \mathscr{B}$ that solves the second of the two equations. We let $Z(Y)$ denote the resulting functional relationship, which is monotonically decreasing. (Monotonicity can be shown by the same argument, following Lemma A5, that we used to prove the monotone dependence of the fixed point of $F_{U}^{\varepsilon}$ on $U$.) We complete the proof by showing that $-Z(\cdot)$ satisfies the assumptions of Blackwell's theorem and therefore has a fixed point in $\mathscr{B}$.

Let $\delta=(1-\varepsilon) /(\rho-\varepsilon) \in(-1,0)$. Since $Z(\cdot)$ is decreasing, it suffices to show that, for any given constant process $x \geqslant 0, Z(Y+x) \geqslant Z(Y)+\delta x$, or, equivalently, $\Delta \geqslant \delta$, where $\Delta=(Z(Y+x)-Z(Y)) / x \leqslant 0$. Let

$$
A_{t}=E_{t}\left[\int_{t}^{T} \exp \left(X_{s}-(1-\varepsilon) Y_{s}+(\rho+1-\varepsilon) Z_{s}(Y)\right) d s\right] .
$$

From the definitions of $Z, \Delta$, and $\delta$, we have

$$
\begin{aligned}
\left(A_{t}+1\right) \exp \left(\Delta_{t} x\right) & =A_{t} \exp \left((\rho-\varepsilon)\left(\Delta_{t}-\delta\right) x+\Delta_{t} x\right)+1 \\
& \geqslant A_{t} \exp \left((\rho-\varepsilon)\left(\Delta_{t}-\delta\right) x+\Delta_{t} x\right)+\exp \left(\Delta_{t} x\right) .
\end{aligned}
$$

Canceling out the term $\exp \left(\Delta_{t} x\right)$ and then the factor $A_{t}$, it follows that $(\rho-\varepsilon)\left(\Delta_{t}-\delta\right) \leqslant 0$, and therefore $\Delta_{t} \geqslant \delta$, completing the lemma's proof.

To complete the existence proof, we need to show that $F_{U}^{1}$ has a fixed point, without assuming that $U$ is bounded from below and above. Suppose first that $U$ is only bounded above, and consider the sequence $U^{n}=\max \{1 / n, U\}, n \in\{1,2, \ldots\}$. Let $V^{n}$ be the fixed point of $F_{U^{n}}^{1}$, shown to exist in Lemma A7. We have argued (with Lemma A6) that $V^{n+1} \leqslant V^{n}$ for all $n$, and therefore $V_{t}=\lim _{n \rightarrow \infty} V_{t}^{n}$ is well defined for all $t \in[0, T]$. Moreover, the bounds of Lemma A5 imply that $V_{t}>0$ for $t<T$. Dominated convergence shows $U$ is a fixed point of $F_{U}^{1}$. For general $U$, we consider the sequence $U^{n}=\min \{n, U\}$, and we apply a similar argument to construct a fixed point of $F_{U}^{1}$. This completes the proof of existence. 
The argument that proves the convexity claims is completely analogous to the case of $A(V)=0$. Where we used the convexity of the function $z \mapsto\|z\|^{2}$, one should now use the convexity of the function $(z, v) \mapsto\|z\|^{2} / v$, for $v>0$, and where we used Lemma A2, one should now use Lemma A6. This completes the proof of Theorem A2.

\section{APPENDIX B}

\section{Proofs Omitted from Main Text}

\section{Proof of Lemma 1}

Let $\Delta_{t}(v)=V_{t}(c+v h)-V_{t}(c), v \geqslant 0$, and

$$
L_{t}(c)=E_{t}\left[\int_{t}^{T} \exp \left(\int_{t}^{s} f_{v}(w) d w\right) f_{c}(s) h_{s} d s\right] .
$$

For any stopping time $\tau$, it follows that

$$
L_{t}(c)=E_{t}\left[\int_{t}^{\tau} f_{c}\left(c_{s}, V_{s}(c)\right) h_{s}+f_{v}\left(c_{s}, V_{s}(c)\right) L_{s}(c) d s+L_{\tau}(c)\right]
$$

while recursion (2) and the gradient inequality imply that

$$
\frac{\Delta_{t}(v)}{v} \leqslant E_{t}\left[\int_{t}^{\tau} f_{c}\left(c_{s}, V_{s}(c)\right) h_{s}+f_{v}\left(c_{s}, V_{s}(c)\right) \frac{\Delta_{s}(v)}{v} d s+\frac{\Delta_{\tau}(v)}{v}\right] .
$$

The proof is completed by applying Lemma C3, with $x=\Delta(v) / v-L(c)$.

\section{Proof of Theorem 1}

The following proof relies heavily on the mathematical results of Appendix A.

Case $1(\gamma=0)$. Given any $c \in \mathscr{C}$, we define the process $U_{t}=\alpha \log \left(c_{t}\right)$. Using the inequality $\exp (|x|) \leqslant \exp (x)+\exp (-x)$ and Jensen's inequality, it follows that $U \in D_{1}^{\exp }$, and by Theorem $\mathrm{A} 1$, there exists a unique pair $(\tilde{V}, Z) \in D_{0}^{\exp } \times D^{n}$ such that

$$
d \tilde{V}_{t}=-\left(U_{t}-\beta \tilde{V}_{t}+\frac{1}{2}\left\|Z_{t}\right\|^{2}\right) d t+Z_{t} d B_{t}, \quad \tilde{V}_{T}=0 .
$$


Defining the new process $V$ to satisfy $1+\alpha V_{t}=\exp \left(\tilde{V}_{t}\right)$ and applying Ito's lemma to the above equation, we obtain

$$
\begin{aligned}
d V_{t}= & -\left(1+\alpha V_{t}\right)\left(\log \left(c_{t}\right)-\frac{\beta}{\alpha} \log \left(1+\alpha V_{t}\right)\right) d t \\
& +\exp \left(\tilde{V}_{t}\right) Z_{t} d B_{t}, \quad V_{T}=0 .
\end{aligned}
$$

The second term of the above expression is a martingale, because

$$
E\left[\left(\int_{0}^{T} \exp \left(2 \tilde{V}_{t}\right)\left\|Z_{t}\right\|^{2} d t\right)^{1 / 2}\right]<\infty
$$

a condition that can be easily confirmed using the Cauchy-Schwarz inequality and the fact that $(\widetilde{V}, Z) \in D_{0}^{\exp } \times D^{n}$. Integrating the expression for $d V_{t}$ from $t$ to $T$ and taking the conditional expectation $E_{t}$, it follows that $V$ solves (2) when $f$ is defined by (8) and $\gamma=0$. Reversing the above argument also shows that $V$ is the unique solution to (2) within the space $\mathscr{V}=\left\{V: 1+\alpha V \in D_{0}^{++}\right\}$. Monotonicity of $V_{0}$ follows from the monotone dependence of $\tilde{V}$ on $U$, part of the conclusion of Theorem A1.

To show concavity, suppose first that $0 \leqslant \alpha \leqslant \beta$. Then $f$ is concave on the domain $\{(c, v): c>0,1+\alpha v>0\}$. (This is just a matter of confirming that $f_{c c}<0$ and $f_{c c} f_{v v} \geqslant f_{c v}^{2}$.) We can then apply Lemma 1 to show a gradient inequality that implies concavity. If $\alpha<0$, then, by Theorem A1, the process $\widetilde{V}$ in the above construction is convex and increasing in $U$, which is in turn convex in $c$. This shows that $1+\alpha V=\exp (\tilde{V})$ is convex in $c$, and therefore $V$ is concave in $c$.

Finally, homotheticity is easily discernible in the ordinally equivalent utilities defined in (9), since, for any $\lambda>0$, we have

$$
\hat{V}(\lambda c)=\hat{V}(c)+\log (\lambda) \frac{1-\exp (-\beta(T-t))}{\beta} .
$$

This can easily be derived from (10) in differential form, which in turn follows from the original recursion (2) and Ito's lemma.

Case $2(\gamma \neq 0)$. Given any $c \in \mathscr{C}$, we define the process $U_{t}=\left(c_{t}^{\gamma} /|\gamma|\right)$ $\exp (-\beta t)$. By Theorem A2 and Lemma A4 (with $p=1+\alpha$ ), there exists a unique $\widetilde{V} \in D_{0}^{++}$such that

$$
\tilde{V}_{t}^{1+\alpha}=E_{t}\left[\int_{t}^{T}(1+\alpha) U_{s} \tilde{V}_{s}^{\alpha} d s\right], \quad t \in[0, T] .
$$


Suppose now that $V$ is related to $\tilde{V}$ by $V_{t}=(|\gamma| / \gamma) \tilde{V}_{t}^{1+\alpha} \exp ((1+\alpha) \beta t)$. Then the above recursion is equivalent to

$$
V_{t} e^{-\beta(1+\alpha) t}=E_{t}\left[\int_{t}^{T}(1+\alpha) \frac{c_{s}^{\gamma}}{\gamma} e^{-(1+\alpha) \beta s}\left|V_{s}\right|^{\alpha /(1+\alpha)} d s\right], \quad t \in[0, T] .
$$

Writing the above as

$$
d\left(V_{t} e^{-\beta(1+\alpha) t}\right)=-(1+\alpha) \frac{c_{t}^{\gamma}}{\gamma} e^{-(1+\alpha) \beta t}\left|V_{t}\right|^{\frac{\alpha}{1+\alpha}} d t+Z_{t} d B_{t},
$$

for some $Z \in D^{n}$, and using integration by parts, it follows that $V$ solves (2) when $f$ is defined by (8) and $\gamma \neq 0$. Letting $\mathscr{V}=\left\{V \in D_{0}: \gamma V>0\right\}$, the above argument shows that $V$ is the unique solution in $\mathscr{V}$. Monotonicity of $V_{0}$ is also a consequence of the above argument and Theorem A2.

To prove concavity of $V_{0}$, we distinguish cases. If $\alpha \gamma>0$, and, as usual, $\gamma<\min \left\{1,(1+\alpha)^{-1}\right\}$, then $f$ is concave on the domain $\{(c, v): c>0$, $\gamma v>0\}$. (This is just a matter of confirming that $f_{c c}<0$ and $f_{c c} f_{v v} \geqslant f_{c v}^{2}$.) Lemma 1 can then be used to show a gradient inequality, which in turn implies concavity. If $\alpha>0$ and $\gamma<0$, then by Theorem A2, $\tilde{V}$ in the above construction is convex and increasing in $U$, which is in turn convex in $c$. It follows that $\gamma V$ is convex in $c$, and therefore $V$ is concave in $c$. If $\alpha<0$ and $\gamma>0$, then $\widetilde{V}$, and hence $V$, is concave and increasing in $U$, which is concave in $c$. This completes the proof of concavity.

Finally, homotheticity is easily discernible in the ordinally equivalent utility defined in (9), since, for any $\lambda>0$, we have $\hat{V}_{t}(\lambda c)=\lambda^{\gamma} \hat{V}_{t}(c)$.

\section{Proof of Lemma 2}

When $\gamma=0$ and $\alpha>0$, or $\alpha, \gamma<0$, the result follows from Lemma 1 . The remaining cases follow (in some cases, using weaker integrability restrictions than that assumed by Lemma 2).

Case $1(\gamma=0$ and $\alpha<0)$. Let $Y_{t}=1+\alpha V_{t}(c), \quad \hat{Y}_{t}=1+\alpha V_{t}(\hat{c}), \Delta c=$ $c-\hat{c}, \Delta Y=Y-\hat{Y}, f(t)=f\left(\hat{c}_{t}, V_{t}(\hat{c})\right)$, and analogously for the partials of $f$. By Lemma A1, we have

$$
\hat{Y}_{t}=E_{t}\left[\exp \left(\int_{t}^{T} \alpha \log \left(\hat{c}_{s}\right)-\beta \log \left(\hat{Y}_{s}\right) d s\right)\right],
$$

and similarly for $Y$. These conditions, together with the gradient inequalities

$$
\alpha \log (c) \geqslant \alpha \log (\hat{c})+\frac{\alpha \Delta c}{\hat{c}}, \quad \text { and } \quad-\beta \log (Y) \geqslant-\beta \log (\hat{Y})-\beta \frac{\Delta Y}{\hat{Y}}
$$


imply

$$
\begin{aligned}
\Delta Y_{t} \geqslant & E_{t}\left[\exp \left(\int_{t}^{T} \alpha \log \left(\hat{c}_{s}\right)-\beta \log \left(\hat{Y}_{s}\right) d s\right)\right. \\
& \left.\times\left(\exp \left(\int_{t}^{T} \frac{\alpha \Delta c_{s}}{\hat{c}_{s}}-\beta \frac{\Delta Y_{s}}{\hat{Y}_{s}} d s\right)-1\right)\right] .
\end{aligned}
$$

Letting $R_{t}=\exp \left(\int_{0}^{t} f_{v}(s)+\beta d s\right)$ and using the fact that $\alpha \log \left(\hat{c}_{s}\right)-$ $\beta \log \left(\hat{Y}_{s}\right)=f_{v}(s)+\beta$ and the inequality $e^{x}-1 \geqslant x$, we obtain

$$
\Delta Y_{t} \geqslant E_{t}\left[\frac{R_{T}}{R_{t}}\left(\int_{t}^{T} \frac{\alpha \Delta c_{s}}{\hat{c}_{s}}-\beta \frac{\Delta Y_{s}}{\hat{Y}_{s}} d s\right)\right] .
$$

Next we define the new probability, $Q$, by letting $d Q / d P=R_{T} / E\left[R_{T}\right]$. Using the change of measure formula for conditional expectations and (B1), we have $E_{t}[d Q / d P]=R_{t} \hat{Y}_{t} / E\left[R_{T}\right]$, and hence

$$
\begin{aligned}
E_{t}^{Q}\left[\int_{t}^{T} \frac{\alpha \Delta c_{s}}{\hat{c}_{s}}-\beta \frac{\Delta Y_{s}}{\hat{Y}_{s}} d s\right] & =\frac{1}{R_{t} \hat{Y}_{t}} E_{t}\left[R_{T}\left(\int_{t}^{T} \frac{\alpha \Delta c_{s}}{\hat{c}_{s}}-\beta \frac{\Delta Y_{s}}{\hat{Y}_{s}} d s\right)\right] \\
& \leqslant \frac{\Delta Y_{t}}{\hat{Y}_{t}}
\end{aligned}
$$

By Lemma $\mathrm{C} 1$, it follows that

$$
\frac{\Delta Y_{t}}{\hat{Y}_{t}} \geqslant \alpha E_{t}^{Q}\left[\int_{t}^{T} e^{-\beta(s-t)} \frac{\Delta c_{s}}{\hat{c}_{s}} d s\right]=\frac{\alpha}{R_{t} \hat{Y}_{t}} E_{t}\left[R_{T} \int_{t}^{T} e^{-\beta(s-t)} \frac{\Delta c_{s}}{\hat{c}_{s}} d s\right] .
$$

Simplifying, we obtain

$$
\begin{aligned}
V_{t}(c)-V_{t}(\hat{c}) & \leqslant \frac{1}{R_{t}} E_{t}\left[R_{T} \int_{t}^{T} e^{-\beta(s-t)} \frac{\Delta c_{s}}{\hat{c}_{s}} d s\right] \\
& =\frac{1}{R_{t}} E_{t}\left[\int_{t}^{T} E_{s}\left[R_{T}\right] e^{-\beta(s-t)} \frac{\Delta c_{s}}{\hat{c}_{s}} d s\right] \\
& =E_{t}\left[\int_{t}^{T} e^{-\beta(s-t)} \frac{R_{s}}{R_{t}} \frac{\hat{Y}_{s}}{\hat{c}_{s}} \Delta c_{s} d s\right] \\
& =E_{t}\left[\int_{t}^{T} \exp \left(\int_{t}^{s} f_{v}(u) d u\right) f_{c}(s) \Delta c_{s} d s\right] .
\end{aligned}
$$


Case $2(\gamma>0$ and $\alpha>0)$. In this case, we show $\mathrm{C}^{\prime}$ under the weaker condition $E\left[\int_{0}^{T-\varepsilon} f_{v}^{2}\left(\hat{c}_{s}, V_{s}(\hat{c})\right) d s\right]<\infty$ for every $\varepsilon \in(0, T)$.

We fix some $h \in F(\hat{c})$ and define $h_{t}^{\varepsilon}=h_{t} 1_{\{t \in[0, T-\varepsilon]\}}$. Concavity of $f$, the gradient inequality, the above restriction on $f_{v}$, and Lemma C3 imply

$$
V_{0}\left(\hat{c}+h^{\varepsilon}\right) \leqslant V_{0}(\hat{c})+\left\langle m(\hat{c}), h^{\varepsilon}\right\rangle, \quad \varepsilon \in(0, T) .
$$

The next step is to take the limit in (B2) as $\varepsilon \downarrow 0$. The first-order conditions (1) and square integrability of $h$ and $\pi$ imply $\lim _{\varepsilon \downarrow 0}\left\langle m(\hat{c}), h^{\varepsilon}\right\rangle=$ $\langle m(\hat{c}), h\rangle$. The proof is completed by showing that $\lim _{\varepsilon \downarrow 0} V_{0}\left(\hat{c}+h^{\varepsilon}\right)=$ $V_{0}(\hat{c}+h)$. The dominated convergence theorem (justified below), continuity of $f$ in its arguments, and $\lim _{\varepsilon \downarrow 0} h^{\varepsilon}=h$, imply

$$
\lim _{\varepsilon \downarrow 0} V_{t}\left(\hat{c}+h^{\varepsilon}\right)=E_{t}\left[\int_{t}^{T} f\left(\hat{c}_{s}+h_{s}, \lim _{\varepsilon \downarrow 0} V_{s}\left(\hat{c}+h^{\varepsilon}\right)\right) d s\right], \quad t \in[0, T] .
$$

If follows from Theorem A2 that $\lim _{\varepsilon \downarrow 0} V_{t}\left(\hat{c}+h^{\varepsilon}\right)=V_{t}(\hat{c}+h)$. The last step is to justify the interchange of limit and expectation. With the uniform (in $\varepsilon$ ) bounds

$$
\hat{c}_{t} / 2 \leqslant \hat{c}_{t}+h_{t}^{\varepsilon} \leqslant 2 \hat{c}_{t}+h_{t}
$$

(because $c+h>0$, we can always rescale $h$ to ensure that the lower bound holds),

$$
0 \leqslant f\left(\hat{c}_{t}+h_{t}^{\varepsilon}, V\left(\hat{c}+h^{\varepsilon}\right)\right) \leqslant f\left(2 \hat{c}_{t}+h_{t}, V(2 \hat{c}+h)\right) .
$$

It is easy to show that $\hat{c} / 2,2 \hat{c}+h \in \mathscr{C}$, which establishes the integrable uniform bounds.

Case $3(\gamma>0$ and $\alpha<0)$. We prove $\mathrm{C}^{\prime}$ under the restriction $E\left[\exp \left(-3 \int_{0}^{T-\varepsilon} f_{v}\left(\hat{c}_{s}, V_{s}(\hat{c})\right)\right) d s\right]<\infty$ for every $\varepsilon \in(0, T)$.

We fix some feasible direction $h \in F(\hat{c})$ and define $h^{\varepsilon}$ as in Case 2. For any $v \geqslant 0$, we define $\Delta_{t}(v)=V_{t}\left(\hat{c}+v h^{\varepsilon}\right)-V_{t}(\hat{c})$ and $\Delta_{t}^{\prime}(0)=\lim _{v \downarrow 0} \Delta_{t}(v) / v$. Below, we prove that the last limit exists, and $\Delta_{0}^{\prime}(0)=\left\langle m(\hat{c}), h^{\varepsilon}\right\rangle$. Inequality (B2) then follows from concavity of $V(c)$ in $c$ and the proof is completed as in Case 2.

Given any $v>0$, by the mean value theorem, we have

$$
\frac{\Delta_{t}(v)}{v}=E_{t}\left[\int_{t}^{T} f_{c}\left(\hat{c}_{s}+\zeta_{s}^{v}, V_{s}(\hat{c}+v h)\right) h_{s}^{\varepsilon}+f_{v}\left(\hat{c}_{s}, V_{s}(\hat{c})+\xi_{s}^{v}\right) \frac{\Delta_{s}(v)}{v} d s\right],
$$


where $\zeta_{s}^{v} \in\left[0, v h_{s}\right]$, and $\xi_{s}^{v} \in\left[0, \Delta_{s}(v)\right]$ (with the convention $[0, a]=$ $[a, 0]$ if $a<0)$. Since $f_{v}+(1+\alpha) \beta<0$, the above linear SDE has the unique solution

$$
\frac{\Delta_{t}(v)}{v}=E_{t}\left[\int_{t}^{T} G_{t, s}^{v} d s\right]
$$

where $G_{t, s}^{v}=\exp \left(\int_{t}^{s} f_{v}\left(\hat{c}_{u}, V_{u}(\hat{c})+\xi_{u}^{v}\right) d u\right) f_{c}\left(\hat{c}_{s}+\zeta_{s}^{v}, V_{s}(\hat{c}+v h)\right) h_{s}^{\varepsilon}$.

To accommodate taking a limit, we derive uniform integrable bounds on $G^{v}$ for small $v$. Monotonicity of $V(c)$ in $c$ and $f_{c c}, f_{c v}<0$ imply that there exists small enough $\bar{v}>0$, such that for all $v \in(0, \bar{v})$,

$$
0 \leqslant f_{c}\left(\hat{c}_{s}+\zeta_{s}^{v}, V_{s}\left(\hat{c}+v h^{\varepsilon}\right)\right) \leqslant f_{c}\left(\hat{c}_{s} / 2, V_{s}(\hat{c} / 2)\right) .
$$

Because the right-hand side of (B3) is proportional to $f_{c}\left(\hat{c}_{s}, V_{s}(\hat{c})\right)$, we have uniform integrable bounds on $G^{v}$ if $E \int_{0}^{T-\varepsilon} f_{c}^{1+\kappa}\left(\hat{c}_{s}, V_{s}(\hat{c})\right) d s<\infty$ for some $\kappa>0$ and every $\varepsilon \in(0, T)$. This condition is implied by the square integrability of $m$, the first-order conditions (1), our assumed restriction on $f_{v}$, and an application of the Hölder inequality. A first implication of these bounds is that $\lim _{v \downarrow 0} \Delta_{t}(v)=0$, and therefore

$$
\lim _{v \downarrow 0} G_{t, s}^{v}=G_{t, s}^{0} \equiv \exp \left(\int_{t}^{s} f_{v}\left(\hat{c}_{u}, V_{u}(\hat{c})\right) d u\right) f_{c}\left(s, \hat{c}_{s}, V_{s}(\hat{c})\right) h_{s}^{\varepsilon} .
$$

A second implication of the bounds on $G^{v}$ is that we can apply the dominated convergence theorem to conclude that $\Delta_{t}^{\prime}(0)=E_{t}\left[\int_{t}^{T} G_{t, s}^{0} d s\right]$, completing the proof.

Case $4(\gamma<0$ and $\alpha>0)$. In this case, we prove $\mathrm{C}^{\prime}$ under the restriction $E\left[\exp \left(2 \int_{0}^{T-\varepsilon} f_{v}\left(\hat{c}_{s}, V_{s}(\hat{c})\right)\right) d s\right]<\infty$ for every $\varepsilon \in(0, T)$.

Let $\hat{V}^{\kappa}$ denote the ordinally equivalent utility process as in (10) but with terminal value $\hat{V}_{T}=\kappa$, where $\kappa \in \mathbb{R}_{-}$. Also, let $\hat{c}_{t}^{\kappa}=\max \left(\hat{c}_{t},|\kappa|\right)$. Then $\hat{V}_{t}(c) \leqslant e^{-\beta(T-t)} \kappa$ and $f_{v}\left(\hat{c}_{t}^{\kappa}, V_{t}^{\kappa}(c)\right)$ is uniformly bounded for any $c \in \mathscr{C}$. We define $\Delta_{t}^{\kappa}(v)=V_{t}^{\kappa}\left(\hat{c}+v h^{\varepsilon}\right)-V_{t}^{\kappa}(\hat{c})$. As in Case 3, we use (B3), $V^{\kappa} \in D_{0}$, and the dominated convergence theorem to show that $\lim _{v \downarrow 0} \Delta_{0}^{\kappa}(v) / v=$ $\left\langle m^{\kappa}\left(\hat{c}^{\kappa}\right), h^{\varepsilon}\right\rangle$ where $m^{\kappa}\left(c^{\kappa}\right)$ is given by (3) with $V^{\kappa}$ taking the place of $V$. Concavity implies

$$
V_{0}^{\kappa}\left(\hat{c}^{\kappa}+h^{\varepsilon}\right) \leqslant V_{0}^{\kappa}\left(\hat{c}^{\kappa}\right)+\left\langle m^{\kappa}(\hat{c}), h^{\varepsilon}\right\rangle, \quad \kappa<0 .
$$

We take the limit as $\kappa \uparrow 0$ to obtain (B2) by using the dominated convergence theorem together with the assumed restriction on $f_{v}$, and the uniform upper bound

$$
m_{t}^{\kappa}\left(\hat{c}^{\kappa}\right) \leqslant \exp \left(\int_{0}^{t} f_{v}\left(\hat{c}_{s}, V_{s}^{0}(\hat{c})\right) d s\right) f_{c}\left(\hat{c}_{t}, V_{t}^{\bar{\kappa}}(\hat{c})\right), \quad \kappa<\bar{\kappa} .
$$


The proof is completed as in Case 2 using the uniform (in $\varepsilon$ ) bounds

$$
f\left(\hat{c}_{t} / 2, V(\hat{c} / 2)\right) \leqslant f\left(\hat{c}_{t}+h_{t}^{\varepsilon}, V\left(\hat{c}+h^{\varepsilon}\right)\right) \leqslant 0 .
$$

\section{Proof of Theorem 2}

We prove Theorem 2 using the results of Theorems 3 and 4 .

Part (a). Under condition I or III, the optimal consumption dynamics are corollaries of Theorems 3 and 4 . (For $\gamma=0$, we obtain $Z=0$, and for $\gamma \neq 0$, we obtain $\alpha Z=0$.)

Part (b). Using the notation of Eq. (17), we have the general identity

$$
\frac{\pi_{s} c_{s}}{\pi_{t} c_{t}}=\exp \left(\int_{t}^{s} \mu_{\tau}^{c}-r_{\tau}-\sigma_{\tau}^{c} \cdot \eta_{\tau} d \tau\right) \frac{\zeta_{s}}{\zeta_{t}},
$$

where $\zeta$ is the exponential supermartingale

$$
\zeta_{t}=\exp \left(\int_{0}^{t}-\frac{1}{2}\left\|\sigma_{\tau}^{c}-\eta_{\tau}^{\prime}\right\|^{2} d \tau+\int_{0}^{t} \sigma_{\tau}^{c}-\eta_{\tau}^{\prime} d B_{\tau}\right) .
$$

Suppose first that $r$ and $\eta$ are deterministic. Then, by part (a) we have

$$
\mu_{t}^{c}-r_{t}-\sigma_{t}^{c} \cdot \eta_{t}=-q_{t}, \quad \text { and } \quad \sigma_{t}^{c}-\eta_{t}^{\prime}=\left(k_{t}-1\right) \eta_{t}^{\prime} .
$$

Since the appropriate Novikov condition is satisfied, it follows that $\zeta$ is a martingale, and therefore

$$
\frac{W_{t}(c)}{c_{t}}=E_{t}\left[\int_{t}^{T} \frac{\pi_{s} c_{s}}{\pi_{t} c_{t}} d s\right]=\int_{t}^{T} \exp \left(-\int_{t}^{s} q_{\tau} d \tau\right) d s .
$$

Next, we consider the case of $\gamma=0$, while $r$ and $\eta$ are potentially stochastic. By Theorem 3, we again have $\mu^{c}-r-\sigma^{c} \cdot \eta=-q$. Given the boundedness of $r$ and $\eta, \zeta$ is a martingale, and the same argument applies. A direct argument that does not rely on Theorem 3, beyond the fact that the unique solution $c$ satisfies the first-order conditions, is as follows. Using the transformation of Lemma A1, we know that the utility process $V=V(c)$ satisfies

$$
\begin{aligned}
1+\alpha V_{t} & =E_{t}\left[\exp \left(\int_{t}^{T} \alpha \log \left(c_{s}\right)-\beta \log \left(1+\alpha V_{s}\right) d s\right)\right] \\
& =e^{\beta(T-t)} E_{t}\left[\exp \left(\int_{t}^{T} f_{v}\left(c_{s}, V_{s}\right) d s\right)\right] .
\end{aligned}
$$


From this equation and (3), it follows that $m_{t}(c)=e^{\beta(T-t)} M_{t} / c_{t}$, where $M$ is the martingale defined by

$$
M_{t}=E_{t}\left[\exp \left(\int_{0}^{T} f_{v}\left(c_{s}, V_{s}\right) d s\right)\right]
$$

The first-order condition $m_{t}(c)=\lambda \pi_{t}$ can be restated as $c_{t} \lambda \pi_{t}=e^{\beta(T-t)} M_{t}$. Using the definition of the wealth process, we obtain $W_{t}(c) \lambda \pi_{t}=$ $\beta^{-1}\left(e^{\beta(T-t)}-1\right) M_{t}$. Dividing the last two equations proves the result.

Part (c). The optimal trading strategy is computed as explained in Section 2.3. Under condition II or condition III, part (b) implies that $\sigma^{W(c)}=\sigma^{c}$. Under condition I or condition III, part (a) implies that $\sigma^{c}=k \eta^{\prime}$. Combining these observations gives the result.

\section{Proof of Lemma 3}

Letting $V$ and $c$ be defined in terms of $X$ and $J$ by (14) and (15), respectively, it suffices to prove that $V_{t}+\int_{0}^{t} f\left(c_{s}, V_{s}\right) d s$ is a martingale. From Eqs. (14) and (15) and the definition of $f$, we have

$$
\frac{\alpha f\left(c_{t}, V_{t}\right)}{1+\alpha V_{t}}=f_{v}\left(c_{t}, V_{t}\right)+\beta=\left((\beta-\alpha) k_{t}-\beta\right) X_{t}+(\alpha-\beta) J_{t}
$$

Using these equations and (4a) in the Ito expansion of (14), we obtain

$$
\frac{\alpha}{1+\alpha V_{t}}\left(d V_{t}+f\left(c_{t}, V_{t}\right) d t\right)=\left[\mathscr{A}_{t} X_{t}+\mathscr{B}_{t}\right] d t+\left(Z_{t}-\left(1-k_{t}\right) \eta_{t}^{\prime}\right) d B_{t}
$$

where $\mathscr{A}_{t}=(\beta-\alpha) k_{t}^{2}-\beta k_{t}-\dot{k}_{t}$, and, with $\mu^{J}$ denoting the drift term of $J$,

$$
\begin{aligned}
\mathscr{B}_{t}= & \mu_{t}^{J}-\left(1-k_{t}\right)\left(r_{t}-\beta+\frac{k_{t}}{2} \eta_{t} \cdot \eta_{t}\right)+k_{t}(\alpha-\beta) J_{t} \\
& +\frac{1}{2} Z_{t} \cdot Z_{t}-\left(1-k_{t}\right) Z_{t} \cdot \eta_{t} .
\end{aligned}
$$

(Hint: In computing $\mathscr{A}$ and $\mathscr{B}$, express everything in terms of $f_{v}$ first, and substitute in the expression for $f_{v}$ last.) We are to show that both $\mathscr{A}$ and $\mathscr{B}$ vanish. In fact, the condition $\mathscr{A}_{t}=0$, together with $k_{T}=1$, is equivalent to (11) (with $\gamma=0$ ), while the condition $\mathscr{B}_{t}=0$, together with $J_{T}=0$, is equivalent to (13). This completes the proof of the lemma. 
Proof of Theorem 3

We have already argued that the unique solution to the first-order conditions is given by (15) for properly selected $\lambda$. (That this solution is an element of $\mathscr{C}$ follows by combining Lemma $\mathrm{C} 1,(15)$, and the assumption that $r$ and $\eta$ are bounded.) Applying Ito's lemma, we obtain

$$
\frac{d c_{t}}{c_{t}}=\frac{d e^{-X_{t}}}{e^{-X_{t}}}+\frac{\alpha d V_{t}}{1+\alpha V_{t}}+\left(\frac{d e^{-X_{t}}}{e^{-X_{t}}}\right)\left(\frac{\alpha d V_{t}}{1+\alpha V_{t}}\right) .
$$

From the dynamics of $X$ in (4a) and Lemma 3 (see Eq. (B4)), we have

$$
\begin{gathered}
\frac{d e^{-X_{t}}}{e^{-X_{t}}}=\left(f_{v}\left(c_{t}, V_{t}\right)+r_{t}+\eta_{t} \cdot \eta_{t}\right) d t+\eta^{\prime} d B_{t}, \\
\frac{\alpha d V_{t}}{1+\alpha V_{t}}=-\frac{\alpha f\left(c_{t}, V_{t}\right)}{1+\alpha V_{t}} d t+\left(Z_{t}-\left(1-k_{t}\right) \eta_{t}^{\prime}\right) d B_{t} .
\end{gathered}
$$

Substituting back into the consumption dynamics and using the identity

$$
\frac{\alpha f\left(c_{t}, V_{t}\right)}{1+\alpha V_{t}}=f_{v}\left(c_{t}, V_{t}\right)+\beta,
$$

the optimal consumption dynamics of Theorem 2 follow immediately.

Optimality verification follows easily from Lemma 2, while uniqueness is a consequence of strict concavity of the utility function.

We proved Eq. (16) as part of Theorem 1, while the optimal trading strategy follows by the argument in Section 2.3, after observing that, by (16), $\sigma^{W(c)}=\sigma^{c}$.

\section{Proof of Lemma 4}

Letting $V$ and $c$ be defined in terms of $X$ and $J$ by (20) and (21), respectively, it suffices to prove that $V_{t}+\int_{0}^{t} f\left(c_{s}, V_{s}\right) d s$ is a martingale. We define $\tilde{\mu}^{J}$ and $\sigma^{J}$ so that

$$
\frac{d J_{t}}{J_{t}}=\tilde{\mu}_{t}^{J} d t+\sigma_{t}^{J} d \tilde{B}_{t}=\left(\tilde{\mu}_{t}^{J}+(1-k) \sigma_{t}^{J} \cdot \eta_{t}\right) d t+\sigma_{t}^{J} d B_{t} .
$$

We can now expand (20) using Ito's lemma, the dynamics of $X$ in (4a), and the identity

$$
\frac{f(c, v)}{v}=\frac{1+\alpha}{\alpha}\left(f_{v}(c, v)+\beta\right)
$$


to obtain

$$
\frac{d V_{t}+f\left(c_{t}, V_{t}\right) d t}{V_{t}}=(1+\alpha k)\left[\mathscr{A}_{t} d t+\left(\sigma_{t}^{J}+\frac{\gamma}{1-\gamma} \eta_{t}^{\prime}\right) d B_{t}\right],
$$

where

$$
\mathscr{A}_{t}=\tilde{\mu}_{t}^{J}+\frac{f_{v}\left(c_{t}, V_{t}\right)}{\alpha}+\frac{\gamma}{1-\gamma}\left(r_{t}+\frac{k}{2} \eta_{t} \cdot \eta_{t}\right)+\frac{\alpha k}{2} \sigma_{t}^{J} \cdot \sigma_{t}^{J}+\frac{1}{1-\gamma} \frac{\beta}{\alpha k} .
$$

We are therefore to show that $\mathscr{A}_{t}=0$. To do so, we begin with the expression

$$
f_{v}\left(c_{t}, V_{t}\right)=\alpha \frac{c_{t}^{\gamma}}{\gamma} V_{t}^{-1 /(1+\alpha)}-(1+\alpha) \beta
$$

Using Eqs. (20) and (21) and simplifying, we observe that the coefficient of $X_{t}$ in the exponent vanishes, resulting in

$$
\frac{f_{v}\left(c_{t}, V_{t}\right)}{\alpha}=\frac{1}{\gamma}(1+\alpha)^{\gamma /(1-\gamma)} J_{t}^{-1}-\frac{1+\alpha}{\alpha} \beta .
$$

Given this expression, the backward SDE (18) (where $Z_{t}=\sigma_{t}^{J} J_{t}$ ) is clearly equivalent to $\mathscr{A}_{t}=0$. Using the above expression for $f_{v}$ in (4a) results in the claimed dynamics for $X$.

\section{Proof of Theorem 4}

Suppose that the assumptions of Theorem 4 are satisfied and that $c$ is given by (21) (as constructed in Section 5.3). The following lemma shows that $c \in \mathscr{C}$.

Lemma B1. The process $\left|J_{t}\right|^{\alpha} \exp \left(\int_{0}^{t} f_{v}\left(c_{s}, V_{s}(c)\right) d s\right)$ is bounded above and away from zero (by deterministic constants, and uniformly in time).

Proof. Fix some $t \in[0, T)$, and let

$$
j_{s}=\gamma J_{s} \exp \left[\int_{t}^{s} a_{u} d u\right], \quad s \geqslant t
$$

where $a_{u}=\gamma(1-\gamma)^{-1}\left[r_{u}-(\beta / \gamma)+(k / 2) \eta_{u} \cdot \eta_{u}\right]$. Then, with $\widetilde{B}$ defined in (12), we have

$$
d j_{s}=-\left[(1+\alpha)^{\gamma /(1-\gamma)} \exp \left(\int_{t}^{s} a_{u} d u\right)+\frac{\alpha k}{2} \frac{z_{s} \cdot z_{s}}{j_{s}}\right] d t+z_{s} d \widetilde{B}_{s}, \quad j_{T}=0 .
$$


By the boundedness of $r$ and $\eta$, we choose a $K>0$ such that $\left|a_{t}\right|<K$ for all $t \in[0, T]$. Using the monotonicity result in Theorem A2, upper and lower bounds for $j_{t}$ are found by replacing $a_{u}$ with $K$ and $-K$, respectively, when $u \geqslant t$. This results in the following bounds for $J_{t}$ :

$$
\frac{1-e^{-K(T-t)}}{K} \leqslant \gamma J_{t}(1+\alpha)^{-\gamma /(1-\gamma)} \leqslant \frac{e^{K(T-t)}-1}{K} .
$$

Using Eq. (B6) and integrating, it follows that, for some constants $C_{1}, C_{2}>0$ that do not depend on the choice of $t$,

$$
C_{1}\left(e^{K(T-t)}-1\right)^{-\alpha} \leqslant \exp \left(\int_{0}^{t} f_{v}(s) d s\right) \leqslant C_{2}\left(e^{K(T-t)}-1\right)^{-\alpha} .
$$

From the same bounds on $\gamma J_{t}$ we also get

$$
C_{3}\left(e^{K(T-t)}-1\right)^{\alpha} \leqslant\left|J_{t}\right|^{\alpha} \leqslant C_{4}\left(e^{K(T-t)}-1\right)^{\alpha},
$$

for some constants $C_{3}, C_{4}>0$ (independent of $t$ ). Multiplying the last two sets of inequalities completes the proof of Lemma B1.

Returning to Eq. (21), and applying Ito's lemma, we obtain the dynamics:

$$
\begin{aligned}
\frac{d c_{t}}{c_{t}}= & -k d X_{t}+\frac{k^{2}}{2}\left(d X_{t}\right)^{2}+\alpha k \frac{d J_{t}}{J_{t}}+\frac{\alpha k(\alpha k-1)}{2}\left(\frac{d J_{t}}{J_{t}}\right)^{2} \\
& -\alpha k^{2}\left(d X_{t}\right)\left(\frac{d J_{t}}{J_{t}}\right) .
\end{aligned}
$$

Simplifying the expression using (18) and the dynamics of $X$ of Lemma 4 results in the theorem's expressions for $\mu^{c}$ and $\sigma^{c}$. Optimality verification follows easily from Lemma 2 using

$$
f_{v}\left(c_{t}, V_{t}\right)=\frac{\alpha}{|\gamma|}(1+\alpha)^{\gamma /(1-\gamma)}\left|J_{t}\right|^{-1}-(1+\alpha) \beta .
$$

The boundedness of $r$ and $\eta$ and the bounds in Lemma A5 imply that for any $\varepsilon>0,\left|J_{t}\right|$ is uniformly bounded away from zero for $t \in[0, T-\varepsilon]$. Uniqueness follows from strict concavity of the utility function.

To determine the optimal consumption and portfolio rules, we use Lemma B2 below in conjunction with Eq. (28) to obtain

$$
W_{t}(c)=(1+\alpha) \gamma V_{t} \exp \left(-X_{t}\right)
$$


Equations (B7), (20), and (21) combined give the claimed optimal consumption-to-wealth ratio. Using expression (B5) (where $\sigma_{t}^{J}=Z_{t} / J_{t}$ ) and (B7), we find

$$
\sigma_{t}^{W(c)}=k \eta^{\prime}+(1+\alpha k) \frac{Z_{t}}{J_{t}}
$$

Arguing as in Section 2.3 we obtain the claimed optimal portfolio allocation.

Lemma B2. For all $t<T$,

$$
\pi_{t} W_{t}(c)=\lambda^{-1}(1+\alpha) \gamma V_{t} \exp \left(\int_{0}^{t} f_{v}(s) d s\right), \quad \gamma \neq 0
$$

Proof. We start with the Ito expansion

$$
\begin{array}{r}
E_{t}\left[V_{T-\varepsilon} \exp \left(\int_{0}^{T-\varepsilon} f_{v}(s) d s\right)\right]-V_{t} \exp \left(\int_{0}^{t} f_{v}(s) d s\right) \\
=E_{t}\left[\int_{t}^{T-\varepsilon} \exp \left(\int_{0}^{s} f_{v}(u) d u\right)\left(V_{s} f_{v}(s)-f(s)\right) d s\right],
\end{array}
$$

for any $\varepsilon \in(0, T)$. In eliminating the martingale part, we have used the fact that $E \exp \left(\int_{0}^{T-\varepsilon} 2 f_{v}(u) d u\right)<\infty$ (which follows from $f_{v} \leqslant 0$ if $\alpha \leqslant 0$ and from the boundedness of $r$ and $\eta$ if $\alpha>0)$ and the fact that $E\left(\int_{0}^{T-\varepsilon} Z_{s}^{\prime} Z_{s} d s\right)<\infty$ for every $\varepsilon \in(0, T)$ (which follows from the Burkholder-Davis-Gundy inequality and the bounds in Lemma A5). Substituting $\gamma\left[V_{s} f_{v}(s)-f(s)\right]=$ $-c_{s}^{\gamma}\left|V_{s}\right|^{\alpha /(1+\alpha)}$ and using the first-order condition

$$
(1+\alpha) \exp \left(\int_{0}^{s} f_{v}(u) d u\right) c_{s}^{\gamma}\left|V_{s}\right|^{\alpha /(1+\alpha)}=\lambda c_{s} \pi_{s}
$$

we obtain, for all $\varepsilon \in(0, T)$,

$$
\begin{aligned}
& \lambda^{-1}(1+\alpha) \gamma V_{t} \exp \left(\int_{0}^{t} f_{v}(s) d s\right) \\
& \quad=E_{t}\left[\int_{t}^{T-\varepsilon} \pi_{s} c_{s} d s\right]+\lambda^{-1}(1+\alpha) \gamma E_{t}\left[V_{T-\varepsilon} \exp \left(\int_{0}^{T-\varepsilon} f_{v}(s) d s\right)\right] .
\end{aligned}
$$

The proof is completed by letting $\varepsilon$ approach zero, provided that we show

$$
\lim _{t \uparrow T} E_{s}\left[V_{t} \exp \left(\int_{0}^{t} f_{v}(s) d s\right)\right]=0, \quad s \in[0, T) .
$$


If $\alpha \leqslant 0$, then $f_{v} \leqslant 0$ and (B8) follows trivially since $\lim _{t \uparrow T} E_{s}\left(V_{t}\right)=0$. If $\alpha>0$, we use (20) and the definition of $X$ to get

$$
V_{t} \exp \left(\int_{0}^{t} f_{v}(s) d s\right)=(\gamma /|\gamma|) \lambda^{1-k} \pi_{t}^{1-k}\left|J_{t}\right|^{1+\alpha k} \exp \left(\int_{0}^{t} k f_{v}(s) d s\right) .
$$

The limit (B8) then follows by Lemma B1.

\section{Proof of Lemma 5}

We first show that $H \leqslant 0$. We have the upper bound for $J$ from (13)

$$
J_{t} \leqslant K-E_{t}\left[\int_{t}^{T} \exp \left(\int_{t}^{s}(\alpha-\beta) k_{u} d u\right)\left(1-k_{s}\right)\left(b+\frac{k_{s}}{2}\left(\Phi^{2}\right)^{\prime} \zeta_{s}\right) Y_{s} d s\right]
$$

for some constant $K$. If $\alpha<0$ then $k_{t}<1$ for $t<T$. The restrictions on $\xi$ along with $Y_{t} \in \mathscr{Y}$ imply that $Y$ has a uniform constant lower bound, which implies a constant upper bound on $J$. This is contradicted, however, if $H_{t}^{i}>0$ for some $i$ since we can consider starting the $Y$ process at time $t$ with $Y_{t}^{i}$ arbitrarily large, violating the upper bound on $J$. It remains to show that $H$ has a finite lower bound. The ODE for $H$ is of the form

$$
\dot{H}_{\tau}=P_{\tau}+Q_{\tau} H_{\tau}+\frac{1}{2} \zeta^{\prime}\left(\Sigma^{\prime} H_{t}\right)^{2}, \quad H_{0}=0,
$$

where $P_{\tau} \in \mathbb{R}^{n}, Q_{\tau} \in \mathbb{R}^{n \times n}$, and $\tau=T-t$ measures time from the horizon date. For any $K \in \mathbb{R}$,

$$
\begin{aligned}
\frac{1}{d \tau} \mathbf{1}^{\prime} e^{-K \tau} H_{\tau} & =\mathbf{1}^{\prime} e^{-K \tau}\left[P_{\tau}+\left(Q_{\tau}-K I\right) H_{\tau}+\frac{1}{2} \zeta^{\prime}\left(\Sigma^{\prime} H_{t}\right)^{2}\right] \\
& \geqslant \mathbf{1}^{\prime} e^{-K \tau}\left[P_{\tau}+\left(Q_{\tau}-K I\right) H_{\tau}\right]
\end{aligned}
$$

Because $Q$ is uniformly bounded, we can choose $K$ large enough so that $\mathbf{1}^{\prime}\left(Q_{\tau}-K I\right) \leqslant 0$, for all $\tau \geqslant 0$, which, combined with $H \leqslant 0$ implies $\mathbf{1}^{\prime} H_{\tau} \geqslant \mathbf{1}^{\prime} \int_{0}^{\tau} e^{K(\tau-s)} P_{s} d s$.

Proof of Lemma 6

The ODE for $F$ is of the form

$$
\dot{F}_{\tau}=P_{\tau}+Q_{\tau}^{\prime} F_{t}+F_{t} Q_{\tau}+2 F_{\tau} \Sigma \Sigma^{\prime} F_{\tau}, \quad F_{0}=0,
$$

where $P_{\tau} \in \mathbb{R}^{n}, Q_{\tau} \in \mathbb{R}^{n \times n}$, and $\tau=T-t$ measures time from the horizon date. The matrix $P$ is negative semidefinite. Lemma 6 then follows from the 
following claim, which can be shown by adapting the proof of Lemma 16.4.2 in Lancaster and Rodman [36] to the variable coefficient case: For any $z \in \mathbb{R}^{n}$,

$$
z^{\prime} F_{\tau} z=\max _{h \text { bounded }}\left\{\int_{0}^{\tau} Z_{s}^{\prime}\left(P_{\tau-s}-2 h_{s}^{\prime} h_{s}\right) Z_{s} d s,\right.
$$

where $Z$ is $\mathbb{R}^{n}$ valued and satisfies $\dot{Z}_{s}=\left(Q_{\tau-s}-2 \Sigma h_{s}\right) Z_{s}, Z_{0}=z$.

\section{APPENDIX C}

\section{Extensions of the Gronwall-Bellman Inequality}

In this appendix we derive some generalizations of the "stochastic Gronwall-Bellman" inequality of Duffie and Epstein [12], results that are used in several of our proofs. The probabilistic setting and notation are the same as in Appendix A. We also use standard lattice notation: $x \wedge y=\min \{x, y\}, x \vee y=\max \{x, y\}, x^{+}=\max \{x, 0\}$.

Lemma C1. Suppose that $\alpha \in D_{1}^{\exp }, \beta \in D_{1}, x \in D_{0}$, and $\varepsilon \in \mathbb{R}$. If $\alpha_{t} \geqslant 0$ and $x_{t} \leqslant E_{t}\left[\int_{t}^{T}\left(\alpha_{s} x_{s}+\beta_{s}\right) d s+\varepsilon\right]$ for all $t \in[0, T]$, then

$$
x_{t} \leqslant E_{t}\left[\int_{t}^{T} \beta_{s} \exp \left(\int_{t}^{s} \alpha_{u} d u\right) d s+\varepsilon \exp \left(\int_{t}^{T} \alpha_{u} d u\right)\right], \quad t \in[0, T] .
$$

The result is also valid with the last two inequalities reversed.

Proof. Let $y_{t}$ represent the right-hand side of the last inequality. It can easily be shown that $y_{t}=E_{t}\left[\int_{t}^{T}\left(\alpha_{s} y_{s}+\beta_{s}\right) d s+\varepsilon\right]$ for all $t$. Letting $\delta=x-y$, it follows that $\delta_{t} \leqslant E_{t}\left[\int_{t}^{T} \alpha_{s} \delta_{s} d s\right]$ for all $t$. Let now $A_{t}=$ $\exp \left(\int_{0}^{t} \alpha_{s} d s\right), C_{t}=\int_{0}^{t} \alpha_{s} \delta_{s} d s$, and $M_{t}=E_{t}\left(C_{T}\right)$. We observe that $M$ is a martingale, and $\delta \leqslant M-C$. It follows that $d\left(A_{t} C_{t}\right)=\alpha_{t} A_{t}\left(C_{t}+\delta_{t}\right) d t \leqslant$ $M_{t} d A_{t}=d\left(A_{t} M_{t}\right)-A_{t} d M_{t}$. Integrating from $t$ to $T$ and applying the operator $E_{t}$ gives $M-C \leqslant 0$, and therefore $\delta \leqslant 0$. The above argument is also valid with all inequalities reversed.

Lemma C2. Suppose that $\alpha^{+} \in D_{1}^{\exp }, \beta \in D_{1}, x \in D_{0}$, and $x$ is rightcontinuous. If for every stopping time $\tau, x_{t} \leqslant E_{t}\left[\int_{t}^{\tau}\left(\alpha_{s} x_{s}+\beta_{s}\right) d s+x_{\tau}\right]$ on $\{t<\tau\}, t \in[0, T]$, and $x_{T} \leqslant 0$, then

$$
x_{t} \leqslant E_{t}\left[\int_{t}^{T} \beta_{s} \exp \left(\int_{t}^{s} \alpha_{u} d u\right) d s\right], \quad t \in[0, T] .
$$

The result is also valid with the last three inequalities reversed. 
Proof. The same argument as in the first part of Lemma C1 shows that it is enough to prove the result when $\beta$ vanishes. Assuming $\beta=0$, suppose that, for some time $t$, the event $A=\left\{x_{t}>0\right\}$ is non-null, and define $\tau=\inf \left\{s \geqslant t: x_{s} \leqslant 0\right\}$. Since $x$ is assumed right-continuous, $x_{\tau} \leqslant 0$, and $x_{s}>0$ on $A \cap\{t \leqslant s<\tau\}$. It follows that, on the event $A \cap\{t \leqslant s<\tau\}$, $x_{s} \leqslant E_{s}\left[\int_{s}^{\tau} \alpha_{u} x_{u} d u\right]$. Therefore,

$$
x_{s} \leqslant E_{t}\left[\int_{s}^{T} \alpha_{u}^{+} 1_{\{u \leqslant \tau\}} x_{u} d s\right] \quad \text { on } A, \quad s \in[t, T]
$$

Applying Lemma $\mathrm{C} 1$ on $A \times[t, T]$, we conclude that $x_{t} \leqslant 0$ on $A$, a contradiction.

A more elaborate argument in the following lemma shows that, for $\beta=0$, Lemma C2 is valid under much weaker integrability assumptions.

Lemma C3. Suppose that $\alpha$ and $x$ are progressively measurable processes satisfying $E\left[\int_{0}^{T} \alpha_{t}^{+} d t\right]<\infty$ and $E\left[\int_{0}^{T} \alpha_{t}^{+} x_{t}^{+} d t\right]<\infty$. Suppose further that $x$ is right-continuous, and, for every stopping time $\tau$,

$$
x_{t} \leqslant E_{t}\left[\int_{t}^{\tau} \alpha_{s} x_{s} d s+x_{\tau}\right] \quad \text { on }\{\tau>t\}
$$

and $x_{T} \leqslant 0$. Then $x_{t} \leqslant 0$ for all $t$.

Proof. Suppose, to the contrary, that, for some time $t$, the event $A=\left\{x_{t}>0\right\}$ is of positive probability. In the argument that follows we restrict time to the interval $[t, T]$. Consider the stopping time $\tau=\inf \left\{s: s \geqslant t, x_{s} \leqslant 0\right\}$. Since $x$ is right-continuous, $x_{\tau} \leqslant 0$, and $x_{s}>0$ on $A \cap\{t \leqslant s<\tau\}$. Moreover, defining the process $X_{s}=E_{s}\left[\int_{s}^{\tau \vee s} \alpha_{u}^{+} x_{u} d u\right]$, $s \in[t, T]$, we have, from $(\mathrm{C} 1)$, that

$$
x_{s} \leqslant X_{s} \quad \text { on }\{s \leqslant \tau\}, \quad \text { for all } s \geqslant t,
$$

and in particular, $X_{t}>0$ on $A$, while of course $X_{\tau}=0$. We also define the stopping times

$$
\tau_{n}=\inf \left\{s: s \geqslant t, X_{s} \leqslant \frac{1}{n}\right\} 1_{A}+t 1_{\Omega \backslash A}, \quad n=1,2, \ldots
$$


and the martingales

$$
M_{s}=E_{s}\left[\int_{t}^{\tau} \alpha_{u}^{+} x_{u} d u\right], \quad N_{s}^{n}=\int_{t}^{s \wedge \tau_{n}} \frac{d M_{u}}{X_{u}}, \quad s \geqslant t, \quad n=1,2, \ldots .
$$

By Ito's lemma,

$$
\begin{aligned}
\log \left(\frac{X_{\tau_{n}}}{X_{t}}\right) & =\int_{t}^{\tau_{n}} \frac{d X_{s}}{X_{s}}-\frac{1}{2}\left(\frac{d X_{s}}{X_{s}}\right)^{2} \geqslant \int_{t}^{\tau_{n}} \frac{d X_{s}}{X_{s}} \\
& =-\int_{t}^{\tau_{n}} \frac{\alpha_{s}^{+} x_{s}}{X_{s}} d s+N_{\tau}^{n}-N_{t}^{n} .
\end{aligned}
$$

Since $X_{t}>0$ on $A$, there exists $\varepsilon>0$ small enough so that the event $\left\{X_{t}>\varepsilon\right\}$ is of positive probability. Fixing such an $\varepsilon$, and using the fact that $X_{\tau_{n}} \leqslant 1 / n$, we obtain

$$
\log (\varepsilon n) \leqslant E\left[\int_{t}^{\tau_{n}} \frac{\alpha_{s}^{+} x_{s}}{X_{s}} d s\right] \leqslant E\left[\int_{0}^{T} \alpha_{s}^{+} d s\right],
$$

where the last inequality follows from (C2). Letting $n$ approach infinity, we reach the conclusion that $E\left[\int_{0}^{T} \alpha_{s}^{+} d s\right]=\infty$, contradicting the lemma's assumptions.

The above lemma is not valid without the assumption $E\left[\int_{0}^{T} \alpha_{t}^{+} d t\right]<\infty$. For example, if $\alpha_{t}=(T-t)^{-1}$, then $(\mathrm{C} 1)$ is satisfied as an equality by $x_{t}=M_{t}(T-t)$ for any martingale $M$.

\section{REFERENCES}

1. F. Antonelli, Backward-forward stochastic differential equations, Ann. Appl. Probab. 3 (1993), 777-793.

2. J. Campbell and L. Viceira, "Consumption and Portfolio Decisions when Expected Returns are Time Varying," working paper, Department of Economics, Harvard University, 1998.

3. S. H. Chew and L. Epstein, The structure of preferences and attitudes towards the timing of resolution of uncertainty, Int. Econ. Rev. 30 (1989), 103-117.

4. S. H. Chew and L. Epstein, Recursive utility under uncertainty, in "Equilibrium Theory with an Infinite Number of Commodities" (A. Kahn and N. Yannelis, Eds.), SpringerVerlag, New York, 1991.

5. J. Cox and C.-F. Huang, Optimal consumption and portfolio policies when asset prices follow a diffusion process, J. Econ. Theory 49 (1989), 33-83.

6. J. Cox and C.-F. Huang, A Variational problem arising in financial economics, J. Math. Econ. 20 (1991), 465-487. 
7. J. Cvitanić, Nonlinear financial markets: hedging and portfolio optimization, in "Mathematics of Derivative Securities" (Dempster and Pliska, Eds.), Proc. of the Isaac Newton Institute, Cambridge Univ. Press, Cambridge, MA, 1997.

8. J. Cvitanić and J. Ma, Hedging options for a large investor and forward-backward SDEs, Ann. Appl. Probab. 6 (1996), 370-398.

9. Q. Dai, J. Liu, and K. Singleton, "Admissibility and Identification of Affine Asset Pricing Models," working paper, GSB, Stanford University, 1997.

10. J. Douglas Jr., J. Ma, and P Protter, Numerical methods for forward-backward stochastic differential equations, Ann. Appl. Probab. 6 (1996), 940-968.

11. D. Duffie, "Dynamic Asset Pricing Theory," 2nd ed., Princeton University Press, Princeton, 1996.

12. D. Duffie and L. Epstein (Appendix with C. Skiadas), Stochastic differential utility, Econometrica 60 (1992), 353-394.

13. D. Duffie and L. Epstein, Asset pricing with stochastic differential utility, Rev. Finan. Stud. 5 (1992), 411-436.

14. D. Duffie, P.-Y. Geoffard, and C. Skiadas, Efficient and equilibrium allocations with stochastic differential utility, J. Math. Econ. 23 (1994), 133-146.

15. D. Duffie and R. Kan, A yield factor model of interest rates, Math. Finance 6 (1996), 379-406.

16. D. Duffie and P.-L. Lions, PDE solutions of stochastic differential utility, J. Math. Econ. 21 (1992), 577-606.

17. D. Duffie, J. Ma, and J. Yong, Black's consol rate conjecture, Ann. Appl. Probab. 5 (1995), 356-382.

18. D. Duffie, M. Schroder, and C. Skiadas, Recursive valuation of defaultable securities, and the timing of resolution of uncertainty, Ann. Appl. Probab. 6 (1996), 1075-1090.

19. D. Duffie, M. Schroder, and C. Skiadas, A term structure model with preferences for the timing of resolution of uncertainty, Econ. Theory 9 (1997), 3-22.

20. D. Duffie and C. Skiadas, Continuous-time security pricing: A utility gradient approach, J. Math. Econ. 23 (1994), 107-131.

21. B. Dumas, R. Uppal, and T. Wang, "Efficient Intertemporal Allocations with Recursive Utility," working paper, 1997.

22. N. El Karoui, S. Peng, and M. C. Quenez, Backward stochastic differential equations in finance, Math. Finance 7 (1997), 1-71.

23. L. Epstein, Behavior under risk: Recent developments in theory and applications, in "Advances in Economic Theory: Sixth World Congress" (J.-J. Laffont, Ed.), Cambridge Univ. Press, Cambridge, U.K., 1992.

24. L. Epstein and Zin, Substitution, risk aversion, and the temporal behavior of consumption and asset returns: A theoretical framework, Econometrica 57 (1989), 937-969.

25. M. Fisher and C. Gilles, "Consumption and asset Prices with Recursive Preferences," working paper, Federal Reserve Board, 1998.

26. L. Foldes, Conditions for optimality in the infinite-horizon portfolio-cum-saving problem with semimartingale investments, Stochastics 29 (1990), 133-171.

27. A. Gelb (ed.), “Applied Optimal Estimation,” MIT Press, Cambridge, MA, 1974.

28. P.-Y. Geoffard, Discounting and optimizing: Capital accumulation as a variational minmax problem, J. Econ. Theory 69 (1996), 53-70.

29. A. Giovannini and P. Weil, "Risk Aversion and Intertemporal Substitution in a the Capital Asset Pricing Model,” NBER working paper No. 2824, Cambridge, MA, 1989.

30. J. M. Harrison and D. Kreps, Martingales and arbitrage in multiperiod securities markets, J. Econ. Theory 20 (1979), 381-408.

31. I. Karatzas, J. Lehoczky, and S. Shreve, Optimal portfolio and consumption decisions for a "Small investor" on a finite horizon, SIAM J. Control Opt. 25 (1987), 1557-1586. 
32. I. Karatzas, and S. Shreve, "Brownian Motion and Stochastic Calculus," Springer-Verlag, New York, 1988.

33. I. Karatzas, and S. Shreve, "Methods of Mathematical Finance," Springer-Verlag, New York, 1998.

34. T. S. Kim and E. Omberg, Dynamic nonmyopic portfolio behavior, Rev. Finan. Stud. 9 (1996), 141-162.

35. D. Kreps and E. Porteus, Temporal resolution of uncertainty and dynamic choice theory, Econometrica 46 (1978), 185-200.

36. P. Lancaster and L. Rodman, "Algebraic Riccati Equations," Oxford Univ. Press, Oxford/New York, 1995.

37. J. Liu, "Portfolio Selection in Stochastic Environments," working paper, Stanford University, 1998.

38. D. Luenberger, "Optimization by Vector Space Methods," Wiley, New York, 1969.

39. J. Ma, P. Protter, and J. Yong, Solving forward-backward stochastic differential equations explicitly - A four step scheme, Probab. Theory Relat. Fields 98 (1994), 339-359.

40. R. Merton, Optimum consumption and portfolio rules in a continuous-time model, J. Econ. Theory 3 (1971), 373-413.

41. R. Merton, "Continuous Time Finance," Basil Blackwell, Oxford, UK, 1990.

42. M. Obstfeld, Risk-taking, global diversification, and growth, Amer. Econ. Rev. 84 (1994), $1310-1329$.

43. E. Pardoux and S. Peng, Adapted solution of a backward stochastic differential equation, Systems Control Lett. 14 (1990), 55-61.

44. S. Pliska, A stochastic calculus model of continuous trading: Optimal portfolios, Math. Oper. Research. 11 (1986), 371-382.

45. M. Schroder and C. Skiadas, "Optimal Consumption and Portfolio Selection with Stochastic Differential Utility," Working Paper No. 226, Dept. of Finance, KGSM, Northwestern University, 1997.

46. M. Schroder and C. Skiadas, "An Isomorphism between Asset Pricing Models with and without Linear Habit Formation," Working Paper No. 247, Dept. of Finance, KGSM, Northwestern University, 1998.

47. C. Skiadas, Recursive utility and preferences for information, Econ. Theory 12 (1998), 293-312.

48. N. Stokey and R. Lucas, "Recursive Methods in Economic Dynamics," Harvard Univ. Press, Cambridge, MA, 1989.

49. L. E. O. Svensson, Portfolio choice with non-expected utility in continuous time, Econ. Lett. 30 (1989), 313-317.

50. J. Wachter, "Portfolio and Consumption Decisions under Mean-Reverting Returns: An Exact Solution in Complete Markets," working paper, Harvard University, 1999.

51. P. Weil, Nonexpected utility in macroeconomics, Quart. J. Econ. 105 (1990), 29-42. 